Rev. Elev. Med. Vet Pays trop, 1964, 17, 3 (441-466)

\title{
La cysticercose bovine en République du Tchad Quelques réflexions sur la situation présente, l'étiologie, le diagnostic, l'immunité et le traitement de cette zoonose
}

\author{
par (M.) GRABER et (M.) THOME \\ (Laboratare de Farcho, Fort-Lamy, Tchad)
}

\begin{abstract}
RÉSUMÉ
10 En République du Tchad, la Cysticercose bovine frappe environ 2 p. 100 des veaux de lait abattus.

Le taux d'ınfestation des bouvilions a considérablement augmenté, passant de 16 p. 100, 1957 à plus de 32 p. 100, 1961-63.

8 à 12 p 100 des adultes sont porteurs de vésicules, sauf au Mayo-Kebbı où le pourcentage atternt 22 p. 100.

$2^{0}$ Les sources d'infestation sant consrituées par les anneaux de Toenia saginato expulsés, les œeufs présents dans les selles des individus atteints et les «œufs anaux » dont le rôle est essentıel dans l'infestation des veaux.

$3^{\circ}$ Les œufs paraissent soumis, pendant 5 mois de l'année, à des conditions extérieures très dures dont les deux principales sont la sécheresse de l'air et la chaleur au sol. Ces facteurs climatiques limitent sans doute la résistance des cufs dans le temps et diminue aınsı les chances d'infestation du bétail.
\end{abstract}

Les auteurs insistent particulièrement sur l'importance des collections d'eau permanente, riches en œufs de $T$. saginato dans la transmıssion de la Cysticercose aux bêtes de boucherie qui se rendent à pied dans les territoires voisıns (R. C. A.) où elles seront abattues.

$4^{\circ}$ Les varıatıons saisonnières dans la fréquence de la Cysticercose bovine n'obéissent à accune règle particulière : elles varıent selon les régions et l'âge des anımaux.

$5^{\circ}$ Le diagnostıc onte mortem de la Cystıcercose esi aléaloire. Les nombreux antigènes employés en Intradermo-réaction donnent des réactions de groupe avec les Cestodes intestinaux et divers Trémalodes. Celte méthode est donc inutilisable dans un pays comme le Tchad où le polyparasitisme est de règle.

$6^{\circ}$ L'immunité conférée par une première contamination paraît solide et semble-t-il, durable, à condition que l'infestation initiale se place assez tard, c'est-à-dire vers le $4^{\mathrm{e}}$ ou le $5^{\mathbf{e}}$ mois de la vie de l'anımal.

$\mathrm{Si}$ l'infestation est trop précoce (de quelques jours à trois mois), la produchion d'anticorps est insuffisante pour neutraliser l'embryon hexacanthe de T. saginato. Des surinfestations ou des réınfestations risquent de se faire jour, avec 
présence simultanée, sur un même anımal, de Cysticerques vivants et de Cysticerques morts.

Un certain nombre d'animaux jouissent d'une ımmuncté naturelle, peut-être innée, le problème n'est pas encore complètement élucidé.

Des essais d'infestation effectués au Laboratoire de FARCHA, ont démontré qu'avant l'âge de deux ans, les deux tiers des bouvillons ont déjà fait ou sont en train de faire une Cysticercose.

Le dernier tiers échappe à l'infection et n'est donc pas immunisé. Cefte façon de voir est confirmée par les chiffres relevés, durant quelques années, à l'abattoir de Bangui (R. C. A.) sur des bovins adultes venus à pied des zones Nord du Tchad (32 p 100 de saisie pour Cysticercose) et par le fait que le taux d'infestation des zones irès touchées (Nord-Cameroun, Mayo-Kebbi) ne dépasse pas 30 p 100.

Dans certains cas, les Cysticerques peuvent vraisemblablement survivre plusieurs années dans les muscles de leur hôte.

$7^{\circ}$ Les différences conslatées entre le Tchad, pays sec et chaud où le taux de Téniasis humain est faible et le Kenya, par exemple, pays beaucoup plus humide ou 30 p. 100 des individus sont porteurs de T.saginata, laissent supposer l'existence en Afrique, de deux types de Cysticercose bovine, dépendant étroitement des facteurs humains et climatiques locaux.

Une carte, 11 tableaux et 109 références biblographiques accompagnent le présent document.

\section{INTRODUCTION}

La Cystıcercose bovine a déjà fait l'objet d'une précédente note (GRABER, 1959) qui, tout en faisant le point de la situation en République du Tchad, résumait la plupart des travaux ayant vu le jour entre 1936 et 1958.

Depuis, un grand nombre d'auteurs se sont penchés sur ce problème quı est d'importance mondiale.

Les études portent particulièrement sur la distribution géographique de la maladie et son Incidence dans certains pays européens tels que l'Allemagne (SCHULTZE-PETZOLD, 1959 ; HERMUS, 1961 ; FREIDRICH, 1961), La Yougoslavie (NENADIC, 1957 ; NENADIC 1958; ZELJKOVIC et BOKOVIC, 1959 ; NENADIC, 1960; NECEV ef ACKOV, 1960; NEVENIC, MEKULI ef RADOVIC, 1960 ; GRUJIC, 1960 ; MIJATOVIC, 1961 ; RUKAVINA, GALL ef DELIC, 1962 ; PAVLICEVIC, 1961 ; MIJATOVIC, 1962 ; LABUDOVIC ef LUKIC, 1963), la Tchécoslovaquie (KOUDELA, 1959), I'U.R. S. S. (MERKUSHEV, ILIN, KOTOVA EI NIKULSHINA, 1962 ; MAMEDOV, 1958 ; ROBSTER ef MALKO, 1961 ; ANDRONIKASHVILI, 1960; PODYAPOLSKAYA, 1960; AVAKYAN, 1961; DAVIDOV, 1963), La Hollande (HOFSTRA, 1959 ; Van KEULEN, 1959 a et b; VINK, 1959), la Suisse (DESPRES et RUOSCH, 1961: DESPRES, 1962) et I'Italie (PELLEGRINI, 1958; PELLEGRINI et BONO, 1957 ; SCHMID, 1958; SGAMBATI, 1959 ; MASELLIS, 1960 ; LEINATI, MARAZZA, GRIMALDI ef PERSIANI, 1963).

En outre, quelques données statistiques viennent d'Afrique (BICHE et THIENPONT, 1959 ; GINSBERG, 1959 et 1960; MARSBOOM, Van PARYS ef BRODSKY, 1960: MC MANUS, 1960; FROYD, 1960: EISA, MUSTAFA ef SOLIMAN, 1962 ; URQUARTH, 1961 ; PEEL, 1961) ef d'Amérique du Sud (LAZZARO, 1961).

Les autres recherches concernent la structure histologique et chimique de Taenia saginata et de Cystircercus bovis (HOLZ et PEZENBURG, 1957 ; MARZUELLO, SQUADRINI ef TAPARELLI, 1957; SILVERMAN ef HULLAND, 1961 ; VOGE, 1960 et 1963), le développement de la forme larvaire et sa dégénérescence (LEE, JONES ef WYANT, 1959 ; GIBSON, 1959 ; MC INTOSH ef MILLER, 1960), l'infestation expérımentale des animaux (Urquarth, 1958, 1959, 1961; FROYD et ROUND, 1959 ; FROYD, 1961).

Quant à la stérilisation des viandes ladres par les rayons $X$, elle est également envisagée en 
Allemagne et en Tchécoslovaquie (RAPIC, BAIC, JEMRIC et MALCIC, 1959 ; PAWEL ef JANICEK, $1963 a$ et $b$ ).

Le but du présent travail est de fournir, dans le cadre de la République du Tchad, et singulièrement de ses zones les plus sèches qui sont les plus riches en bétail, un certain nombre de renseignements intéressant la situation actuelle de la cystıcercose bovine, l'étiologie de la maladie, son diagnostıc, la résistance des animaux au parasite ef le traitement à recommander.

\section{I. - SITUATION ACTUELLE}

\section{A. - Les Veaux}

Les chiffres ne concernent que l'abattoir de Fort-Lamy où sont enregistrés les plus gros abattages de veaux originaires des zones de Massakory-Moussoro (Tableau I.

TABLEAU NO I

\begin{tabular}{|c|c|c|c|}
\hline Années & $\begin{array}{c}\text { Veaux } \\
\text { abattus }\end{array}$ & $\begin{array}{c}\text { Veaux } \\
\text { parasités }\end{array}$ & Pourcentages * \\
\hline 1958 & 176 & 1 & 0,56 \\
1959 & 97 & 1 & 1 \\
1960 & 177 & 3 & 1,69 \\
1961 & 254 & 2 & 0,78 \\
1962 & 634 & 12 & 1,89 \\
1963 & 1.039 & 7 & 0,67 \\
\hline
\end{tabular}

* Chiffres aimeblement communiqués par le Dr. TROUETTE, Directeur des abattoirs de Farcha.

Les jeunes proviennent dırectement d'élevages locaux ou sont achetés par des sociétés de boucherie et engraissés avant l'abattage. Ces chiffres ne sont valables que pour l'Ovest tchadien. Dans d'autres régions (Ouaddaï et Mayo-
Kebbi) les taux d'infestation paraissent bien supérieurs, si l'on s'en tient à des résultats fragmentaires glanés çà ef là.

$$
\text { B. - Bouvillons (Tableau II) }
$$

TABLEAUU II II

\begin{tabular}{|c|c|c|c|c|c|c|}
\hline \multirow[b]{2}{*}{ Régions } & \multicolumn{3}{|c|}{ Années 1954 - 1958} & \multicolumn{3}{|c|}{ Années 1959 - 1963} \\
\hline & $\begin{array}{l}\text { Animatux } \\
\text { abattus }\end{array}$ & $\begin{array}{l}\text { Animaux } \\
\text { perasités }\end{array}$ & Pourcentage & $\begin{array}{l}\text { Animanx } \\
\text { abattus }\end{array}$ & $\begin{array}{c}\text { Animaur } \\
\text { perasités }\end{array}$ & Pourcentage \\
\hline Kanem & 450 & 67 & 14,8 & & & \\
\hline Chari-Baguirimi & 462 & 74 & 16 & 288 & 92 & . 31,9 \\
\hline Batha & & & & 55 & 20 & 36.3 \\
\hline
\end{tabular}


Ce qui frappe, au vu de ce tableau, c'est l'augmentation sensible du nombre d'anımaux de 8 à 22 mois porteurs de cystıcerques, dans les régıons Ouest du Tchad et surtout dans le ChariBaguirmi, Le pourcentage d'animaux atteints passe du simple au double de 1958 à 1963. II' n'y $a_{1}$ pour l'instant, guère d'explication valable : les investigations se poursuivent.

C. - Animoux de boucherie odulfes (Tableau III)

TABLEAUU IO III

\begin{tabular}{|c|c|c|c|c|}
\hline \multirow[b]{2}{*}{ Régiong } & \multicolumn{3}{|c|}{$1959-1963$} & \multirow{2}{*}{$1952-1959$} \\
\hline & $\begin{array}{l}\text { Animaux } \\
\text { eutopstés }\end{array}$ & $\begin{array}{c}\text { Animaux } \\
\text { Darasités }\end{array}$ & Pourcentages & \\
\hline $\begin{array}{l}\text { Fort-Lamy } \\
\text { (abattoirs) }\end{array}$ & 1.330 & 16 & 1,2 & $0,4-0,6$ \\
\hline Chari-Baguirnoi & 89 & 11 & 12,3 & \\
\hline Kanem & 133 & 11 & 8,2 & \\
\hline Bathe & 315 & 34 & 11 & \\
\hline Ouadda: & 641 & 65 & 10,1 & 8 à 14 \\
\hline Moyen-Cherry & 202 & 18 & 11,2 & 5 à 12 \\
\hline Marro-Kebbi & 268 & 57 & 21,2 & \\
\hline
\end{tabular}

Plusieurs remarques s'ımposent :

a) Les adultes sont trois fois moins parasités que les bouvillons : le même fait a été signalé en Sierra-Leone (PEEL, 1953 ef 1961), mais its le sont autant que les veaux, si l'on s'en tient aux chiffres de l'abattoir de Fort-Lamy.

b) Sur dix ans, le nombre d'anımaux infestés semble assez constant, 51 l'on compare les chiffres du tableau no lil à ceux qui ont été fournis précédemment (GRABER, 1959).

L'inspection de routine, dans les abattoirs de brousse, permet une meilleure recherche des cysticerques, car les épaules sonł levées * et des coupes sont autorisées, notamment dans les psoas, la langue, le cour et les masséters, zones où se trouvent localisés de préférence les parasites, comme le constatent en Afrique du Sud VAN DEN HEEVER et REINECKE (1963).

* Elles ne le sont pas à l'abattoir de Fort-Lamy, c) Il a été établı une distinction très nette entre les chiffres donnés par l'abattorr de Fort-Lamy et ceux obtenus à partir d'animaux âgés achetés en divers points du Chari-Baguirmi. Les pourcentages d'infestations sont différents : 1,2 p. 100 dans le premier cas et environ 12 p. 100 dans le second.

Ce faif est, de prime abord, paradoxal. II s'explıque assez bien si l'on consıdère l'origine du bétal tué aux abattoirs de Fort-Lamy. II s'agit, dans la majorité des cas d'animaux de ranch ou d'animaux nomades, de préférence des mâles de 6 à 9 ans spécialement choisis pour l'exportation et dans l'ensemble, assez peu parasités. En effet, une enquête effectuée en 1963 dans la région d'Atı (Centre-Tchad) et portant sur 239 têtes a démontré que le bétail nomade de cette région héberge deux fois moins de cysticerques que le bétail sédentaire. La même constatatıon a été faite en août 1963 au Kanem. 
Par contre, si l'on s'adresse uniquement à du bétail sédentaıre, constitué surtout par de vieilles vaches, comme c'est le cas pour les animaux du Chari-Baguirmi tués entre 1961 et 1963 au laboratoire de Farcha, on retrouve, à peu de choses près, le taux d'infestation constaté ailleurs (Carte no 1).

d) Si I'on étudie la carte jointe au présent document, le pourcentage de Cysticercose semble être sensiblement le même dans toutes les régions admınıstratives du pays, sauf au Mayo-Kebbı où il se situe autour de 21 p. 100. La zone de Bongor, fortement peuplée, se rattache d'ailleurs, du point de vue cysticercose à celle du Nord-Cameroun où près de $30 \mathrm{p} .100$ de bovins sont parasıtés (MAROUA, 1963).

Le Moyen-Chari (Fort-Archambault), bien que jouissant d'un climat soudano-guinéen, ne se

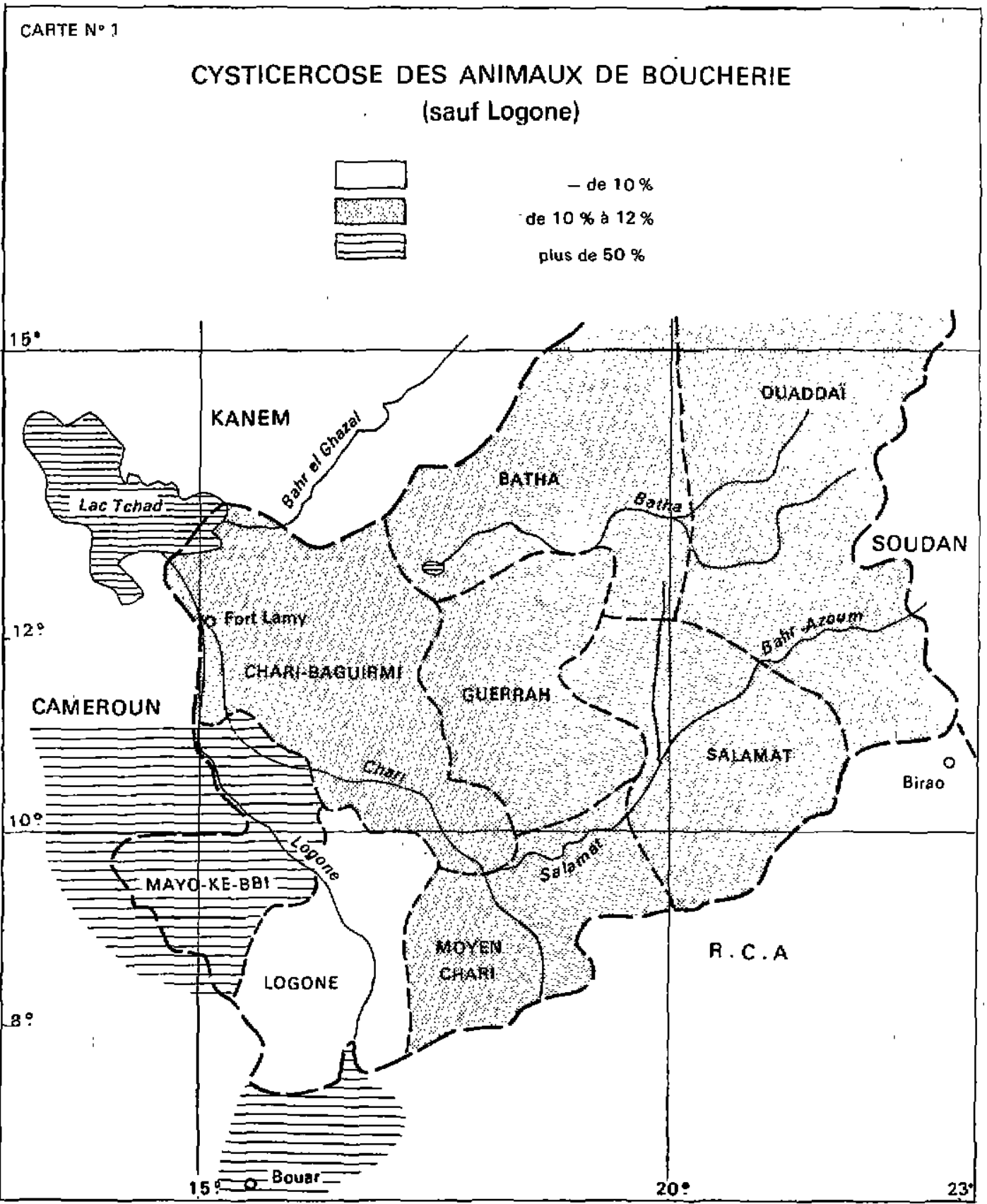


distingue pas du reste du pays, l'approvisionnement en bétail de boucherie étant assuré par des animaux venus du Ouaddaï ou du Batha et par des troupeaux transhumants.

Les renseignements sur l'état de la cysticercose dans les pays voisins du Tchad ne sont pas abondants : outre ce qui vient d'être dit à propos du Nord-Cameroun, les taux d'infestation paraissent voisins de 10 p. 100 dans le Nord de la Nigeria (GRABER, 1959) et de 11 p. 100 dans le Sud du Soudan (EISA, MUSTAFA et SOLIMAN, 1962), ce qui semblerait indıquer qu'entre Kano et Port-Soudan, toute cette portion d'Afrique située en bordure du désert est soumise aux mêmes conditions.

Plus au Sud, en R. C. A., les bovins ladres représentent 30 à 50 p. 100 des animaux tués aux abattoirs de Bangui et de Bouar. Ces chiffres sont bien supérieurs à ceux du Tchad.

Des pourcentages assez semblables ont été relevés ces dernières années en divers points d'Afrique noire (Tableau IV).

TABLEAD N N $T$

\begin{tabular}{|c|c|c|c|}
\hline Pays & Annéeg & Pourcentages & inteurs \\
\hline \multirow[t]{3}{*}{ Kenya } & .1956 & 30 & $\begin{array}{l}\text { Ginsberg, Cameron, } \\
\text { Godard ot Grieve }\end{array}$ \\
\hline & 1958 & 39 ì 44 & Mc. Owan \\
\hline & 1960 & 31 & Froyd \\
\hline \multirow[t]{2}{*}{ Uganda } & $195^{\circ}$ & $10 \mathrm{~B} 25$ & Repport annuel \\
\hline & 1960 & 20 ̀े 30 & Rollingon \\
\hline \multirow[t]{3}{*}{ Ruanda-Burundi } & 1949 & 80 & Fajin et de Ramée \\
\hline & 1959 & 69 & Biche et Thienpont \\
\hline & 1960 & 80 & $\begin{array}{l}\text { Mesboom, Van Parys, } \\
\text { et Brodsky }\end{array}$ \\
\hline Sierra-Léone & 1961 & 38,4 & Peel \\
\hline
\end{tabular}

La lecture de ces statistiques révèle que la Cysticercose bovine prend, en Afrique, un visage différent, selon que l'on s'adresse d'une part à des régions sèches comme le Tchad ou d̀ des zones plus humides comme la R. C. A., l'Uganda ou la Sierra-Leone, d'autre part à des pays où le peuplement humain est déjà important (RuandaBurundi).

\section{II. - DONNÉES ÉTIOLOGIQUES}

Les considérations générales, valables pour le Tchad, ont déjà été exposées antérieurement (GRABER, 1959). Un certain nombre de points méritent cependant d'être précisés :

\section{a) Les Sources d'infestation du bétail :}

Le premier problème concerne le nombre d'anneaux et d'œufs émis par les porteurs humains de Taenia saginata, problème fort controversé qui est résumé dans le tableau no $\mathrm{V}$.
L'évacuation des anneaux de Taenia et des œufs est sujette à de sensibles variations ef l'on observe de grandes différences journalıères qui dépendent de l'individu plus que du Cestode.

Chez l'anımal, c'est ce qui se passe également avec Moniezıa expansa du mouton ou Raillietina tetragona du poulet.

Le nombre d'œufs renfermés dans les anneaux est sous la dépendance de divers facteurs dont certanns sont liés aux contractions qui agitent le proglottis mûr détaché dans la lumière intestinale.

Au niveau de la zone de rupture, de nombreux œufs, du fait des contractions, sont expulsés et se retrouvent dans les matières fécales (DOBY, DOBY-DUBOIS et DEBLOCK, 1957).

En quittant le tractus digestif, le proglottis force le sphincter anal et subit de grosses compressions qui ont pour conséquence le dépôt d'un grand nombre d'œufs sur les marges de l'anus 


\begin{tabular}{|c|c|c|c|}
\hline Luteurs & Annóes & $\begin{array}{c}\text { Nombre d'anneaux } \\
\text { fmia }\end{array}$ & $\begin{array}{l}\text { Nombre d'oeufs } \\
\text { par enneaux }\end{array}$ \\
\hline $\begin{array}{l}\text { Cobbold, elté par } \\
\text { Parlier. }\end{array}$ & 1938 & 400 par an & 30.000 \\
\hline Raililet & 1893 & & 8.000 \\
\hline $\begin{array}{l}\text { Bochert, cité par } \\
\text { Talavera }\end{array}$ & 1957 & & 80.000 \\
\hline $\begin{array}{l}\text { Penfold, Penfold et } \\
\text { Philips }\end{array}$ & 1937 & 8 par jour & \\
\hline$S_{1}$ Irerman & 1954 & $\begin{array}{c}\text { de } 0 \text { a } 12 \text { par } \\
\text { Jour }\end{array}$ & \\
\hline Nomis & 1956 & & 100.000 \\
\hline Urquarth & 1961 & & $120 \geq 33.000$ \\
\hline Errshov & 1956 & $\begin{array}{l}28 \text { par jour } \\
20 \text { jours chaque mo1s }\end{array}$ & . \\
\hline Graber & 1959 & & 10.000 environ \\
\hline
\end{tabular}

(MAZOTTI, 1944, RIJPSTRA, SMIT et SWELLENGREBEL, 1961).

L'anneau rejeté à l'extérieur sera donc plus ou moins riche en œufs dont le nombre est fonction des mouvements du parasite dans la lumière intestınale et des pressions qui s'exercent sur luı, au niveau du sphincter anal, au moment de son élimination.

I1. en résulte qu'un porteur de Taenia saginato est dangereux de trois façons:

par les cufs émis dans les selles.

par les anneaux évacués.

par les ceufs collés aux marges de l'anus.

D'après URQUARTH (1961), le nombre d'œufs dans les selles serait apparemment le même que dans les anneaux.

Selles et proglottis constituent les sources d'infestation les plus classiques pour le bétail qui vit en symbiose avec l'homme.

Les « œufs anaux » sont capables, vu les règles d'hyglène sommaires en usage dans certains pays africaıns, de passer sur les doigts et sous les ongles des personnes parasitées et d'y demeurer aussi longtemps qu'ils n'en seront pas chassés par un nettoyage sérieux. Si le porteur doit s'occuper d'un troupeau, il risque, au moment de la traite, de déposer sur les poils et sur les tétınes des femelles quelques œufs de Taenia susceptıbles d'infester par la suite la mère et son veau.
Ailleurs, c'est en obligeant les jeunes à boire au seau que I'on communique la cysticercose (URQUARTH, 1961).

Cette notion d'«œufs anaux» méritait de retenir l'attention : elle est, en partie, à l'origine de la contamination des animaux producteurs de lait et de leur descendance.

\section{b) Modalités d'infestation des animaux :}

1) La contamination se produit non seulement au stade élevage, mais encore au stade commercialisation.

On sait que, bien souvent en Afrique, les bêtes de boucherie sont achetées dans les zones de production, rassemblées en troupeaux et acheminées à pied vers les centres de consommation quelquefors sur des distances considérables. De véritables «routes du bétaii » se sont ainsı créées, jalonnées de villages et de points d'eau où les animaux se reposent au cours du voyage. Or, les points d'eau qui sont souvent des mares permanentes doivent être incrimınés' au premier chef, car ils semblent particulièrement riches en ceufs de Taenio saginato libérés par des individus afteints, au moment aù ils se baignent ou lorsqu'ils lavent leurs vêtements.

Le bétail de passage en profite alors largement.

C'est arnsi que l'abattoir de Bangui en R. C. A. est approvisionné pour les trols quarts par des zébus provenant du Tchad, c'est-à-dıre des 
marchés du Ouaddal, du Batha et du Salamat. Les troupeaux constitués dans la région de FortArchambault descendent à pied et par petites étapes vers Bangui. La distance représente environ $600 \mathrm{~km}$.
Au départ, si l'on en croit les statistiques du Tchad, le toux d'infestation par Cysticercus bovis oscille autour de 10-11 p. 100. A l'abattoir de Bangui, il va de 25 à' 30 p. 100 (tableau'no VI).

TABLEFAU NO VI

\begin{tabular}{|c|c|c|c|c|}
\hline \multirow{2}{*}{ Années } & \multicolumn{2}{|c|}{$\begin{array}{l}\text { Nombre d'animaux } \\
\text { Inspectés }\end{array}$} & \multicolumn{2}{|c|}{ Taux c'unfestation } \\
\hline & Tehad & R.C.A. & Tched & Tchad + R.C.A. \\
\hline 1954 & 10.307 & & 30 & \\
\hline 1955 & 6.929 & 1.696 & 25 & 28 \\
\hline 1956 & 8.807 & 2.209 & 28 & 29 \\
\hline 1957 & 8.786 & 2.603 & 30 & 31 \\
\hline 1958 & 10.307 & 3.093 & 30 & 28 \\
\hline & \multicolumn{2}{|c|}{ Tchad + R.C.A. } & & \\
\hline 1959 & \multicolumn{2}{|c|}{12.100} & & 29 \\
\hline 1960 & \multicolumn{2}{|c|}{16.500} & & 25 \\
\hline 1961 & \multicolumn{2}{|c|}{16.300} & & 27 \\
\hline
\end{tabular}

Pour les années 1959, 1960 ef 1961, nous ne possédons que des statistiques groupées comprenant à la fois le Tchad et la R. C. A. Il est donc difficile de définır ce qui revient aux animaux de l'un ou l'autre territorre. Cependant, comme les taux d'infestation globaux ne semblent pas, durant cette période, présenter des modifications très sensibles, il est permıs de penser que le nombre d'animaux parasités originaires du Tchad demeure approximativement le même que pendant les années 1954-58.

La différence entre les pourcentages enregistrés au Tchad et en R. C. A. (13 d̀ 20 p. 100) représente l'infestation acquise en cours de route, ce que confirment les observations faites en R. C. A. en 1963 : lorsque cette source d'infestation est tarie, c'est-à-dire quand les anımaux sont transportés rapidement par camion de FortArchambault à Bangui, le pourcentage d'infestation tombe alors à 14 p. 100 .

Les routes du bétail traditionnelles paraissent donc constituer pour les animaux de boucherie, une source d'infestation non néglıgeable, et, dans certaines circonstances, le nombre d'ani- maux atteints peut doubler, même sur une distance relativement courte. Le même phénomène a été observé por BIRKETT (1953) en SierraLeone.

2) Facteurs défavorables: ce sont surtout des facteurs physiques. Ils interviennent en limitant les possibilités d'infestation. A cet égard, le rôle de la chaleur et de la sécheresse ne doit pas être sous-estimé au Tchad.

JEPSEN et ROTH (1949), au cours de l'été 1947 qui fut particulièrement $\mathrm{sec}$, placent des cufs et des anneaux de $T$. saginata sur des touffes d'herbe qu'ils laissent à l'air libre. Plus le temps d'exposition du matériel infestant est long, plus le nombre de Cysticerques, mis en évidence à l'autopsie de veaux expérimentalement infestés, diminue. Les auteurs pensent que la dessiccation assure la destruction d'un grand nombre d'œufs.

Cette opinion est partagée par SILVERMAN $(1956, b)$ qui fixe à 14 jours la survie maxımum des ceufs, s'il n'y a pas une humidité de surface suffisante. LUCKER et DOUVRES (1960) sont du même avis. 
La chaleur joue un rôle semblable : les cuts sont détruits en 10 minutes à $59^{\circ} \mathrm{C}$. Ils sont susceptibles d'être réactivés quatre heures après avoir été chauffés à 45० C (SILVERMAN, 1956, b). Or, au Tchad, des températures de $48-50^{\circ} \mathrm{C}$, au ras de sol, sur un terrain sablonneux et en plein soleil, ne sont pas rares de février à juin. Ces maxima se situent entre 11 et $13 \mathrm{~h}$. Le degré hygrométrique est alors inférieur à 15 p. 100. A l'ombre, aux mêmes époques de l'année, la température varie de 37 à $42^{\circ} \mathrm{C}$. De plus, des vents violents, soufflant de l'Est, dessèchent la surface du sol. Dans ces conditions, on peut donc penser que, pendant la saison sèche, beaucoup d'œufs de Toenia saginata disparaissent et qu'un petit nombre d'entre eux seulement est capable d'infester les anımaux.

De juillet à décembre, les températures sont, dans l'ensemble, plus basses ef le degré hygrométrique plus élevé : la résistance des œufs est alors plus grande. Le fait est bien connu dans les pays où l'humidité est importante : les œufs demeurent intacts de 2 à 6 mois (THORNTON, 1949; CHANDLER, 1956 ; LAPAGE, 1956). Au Kenya, DUTHIE ef VAN SOMEREN (1948) avancent même le chiffre d'un an.

Quant aux milieux humides (mares, collections d'eau, etc...). Ils sont favorables à la conservation ef à la survie des œufs (JEPSEN ef ROTH, 1947 ; SILVERMAN, 1956 'b ; PEEL, 1961).

Au Tchad, à l'exception des zones où subsistent des mares permanentes, l'influence des agents extérieurs, chaleur ef degré hygrométrique, semble diminuer, une bonne partie de l'année, les chances d'infestation du bétail. Par contre, dans les pays plus humides et moins chauds, c'est l'inverse qui paraît se produire.

c) A quelle époque de l'onnée, l'infestation du bétail par les cysticerques est-elle maximum?

Quelques renseignements on tété recueillis dans l'Ouest, le Centre ef l'Est tchadien : (Tableau VII ef V|il).

TABLEAU No VII. -- Ouest Tchadien - Bouvillons de 6 à 22 mois

\begin{tabular}{|c|c|c|c|}
\hline Hois et amoée & $\begin{array}{c}\text { Pourcentase d'animaux } \\
\text { parasités }\end{array}$ & Mois et année & $\begin{array}{c}\text { Pourcentage d'amimaux } \\
\text { peragitéa }\end{array}$ \\
\hline $\begin{array}{ll}\text { Jarrier } & 1955 \\
& \\
\text { Mars } & 1955 \\
\text { Avril } & 1955 \\
\text { Mai } & 1955 \\
\text { Juin } & 1955 \\
\text { Juillet } & 1955 \\
\text { Août } & 1955 \\
\text { Septembre } & 1955 \\
\text { Octobre } & 1955\end{array}$ & $\begin{array}{r}28 \\
15 \\
25 \\
10 \\
5 \\
10 \\
0 \\
4 \\
0\end{array}$ & $\begin{array}{ll}\text { Janvier } & 1957 \\
\text { Février } & 1957 \\
\text { Mars } & 1957 \\
\text { Avril } & 1957 \\
\text { Mail } & 1957 \\
\text { Juin } & 1957 \\
& \\
\text { Aoflt } & 1957 \\
\text { Septembre } & 1957 \\
\text { Octobre } & 1957 \\
\text { Horembro } & 1957 \\
\text { Décembre } & 1957\end{array}$ & $\begin{array}{r}22 \\
12 \\
0 \\
20 \\
0 \\
11 \\
\\
16 \\
20 \\
8 \\
17 \\
16\end{array}$ \\
\hline
\end{tabular}

Apparemment, les résultats ne coïncident pas avec ce qui a été sıgnalé dans les deux paragraphes précédents. Peut-être ainsi qu'ıl sera dit plus loin' faut-il incriminer la longévité propre des parasites, les infestations, se faisant à des époques différentes et la dégénérescence des cysticerques étant sujette à de nombreux aléas.

La lecture de ces chiffres permet de constater simplement que chez les bouvillons, les cysticerques sont présents toute l'année avec un léger creux au printemps.
Chez les adultes, l'ınfestation mınımum se situe en automne dans l'Est tchadien, et au printemps dans le centre. A Fort-Lamy par contre, la cysticercose est surtout une affection d'hivernage (de juın à septembre).

La littérature est pauvre en travaux faisant état de la distribution saisonnière des cysticerques (: KOUDELA (1959), en Tchécoslovaquie, signale que la Cysticercose bovine passe par un maximum en septembre pour toucher le minimum en fin d'année. A Trieste (SCHMiD, 1958), le point culminant est atteınt en juin. 
TABLFAU N O VIII - AduItos

\begin{tabular}{|c|c|c|c|c|c|}
\hline \multicolumn{2}{|c|}{ Ouaddar(Abecher) } & \multicolumn{2}{|c|}{ Bathy (At1) } & \multicolumn{2}{|c|}{ Chari-Baguimini (F.L.) } \\
\hline Mois et ammée & $\begin{array}{l}\text { Pourcentage d'ani- } \\
\text { maur parasités. }\end{array}$ & Mois et année & $\begin{array}{l}\text { Pourcentage d'ant- } \\
\text { maux parasités. }\end{array}$ & Mois et année & $\begin{array}{l}\text { Pourcentage d'ani- } \\
\text { maux perasi tés. }\end{array}$ \\
\hline $\begin{array}{ll}\text { Avril } 1959 \\
\text { Mai } 1959 \\
\text { Juillet-Août1959 } \\
\text { Septembre } 1959 \\
\text { Detobre } & 1959 \\
\text { Hovembre } & 1959 \\
\text { Décembre } & 1959 \\
\text { Janrler } & 1960 \\
\text { Février } & 1960 \\
\text { Mara } & 1960\end{array}$ & $\begin{array}{r}16 \\
10 \\
10 \\
3 \\
0 \\
5 \\
9 \\
5 \\
13 \\
10\end{array}$ & $\begin{array}{ll}\text { Janvier } & 1959 \\
\text { Février } & 1957 \\
\text { Mars } & 1957 \\
\text { Avril } & 1957 \\
\text { Mai } & 1957 \\
\text { Juin } & 1957 \\
\text { Juillet } & 1957 \\
\text { Septembre } & 1957 \\
\text { Octobre } & 1957 \\
\text { Novembre } & 1957 \\
\text { Décembre } & 1957\end{array}$ & $\begin{array}{r}22 \\
12 \\
0 \\
10 \\
0 \\
11 \\
16 \\
20 \\
8 \\
17 \\
16\end{array}$ & $\begin{array}{ll}\text { Août } 1959 \\
\text { Sept.1959 } \\
\text { Octobre } 1959 \\
\text { Novemb. } & 1959 \\
\text { Dścemb. } & 1959 \\
\text { Janvier } & 1960 \\
\text { Fóvrier } & 1960 \\
\text { Mara } & 1960 \\
\text { Avril } & 1960 \\
\text { Mai } & 1960 \\
\text { Juin } & 1960 \\
\text { Juillet } & 1960 \\
\text { Aoút } & 1960\end{array}$ & $\begin{array}{c}3,2 \\
0,7 \\
0 \\
0 \\
0 \\
0 \\
0 \\
0 \\
0 \\
7,3 \\
3 \\
3 \\
1\end{array}$ \\
\hline
\end{tabular}

\section{III. - DIAGNOSTIC}

\section{A. - Ante-mortem}

Afin de compléter et de renforcer le contrôle sanıtaıre, il serait intéressant de savoir si, à l'achat, l'animal est porteur de cysticerques. Si c'est le cas, Il sera alors utilisé solt sous forme de viande séchée ou de jus de viande, soit mis en conserve.

En matière de Cestodes, la méthode consiste à rechercher, chez les individus suspects, les anticorps liés à la présence du parasite. En médecine humaine, diverses réactıons sérologiques (Dévıation du complément, précipitation, etc...) ou allergiques ont été préconisées. L'antigène est préparé à partir des Cestodes eux-mêmes ou de leurs cysticerques. Divers auteurs ont expérimenté Cysticercus tenuicollis (MORENAS, 1933), la vésıcule totale de Cysticercus cellulosae (TRAWINSKI, 1947, 1957 ; BIAGI et TAY, 1958), le liquide du kyste hydatique (CULBERSTON, 1941), mauvals antigène auquel on préfère les scolex d'Echinococcus.

Récemment, BRISOU (1946) recommande l'antigène «Ténia » préparé à partır des Cestodes complets appartenant aux espèces, suivantes: Toenia saginata, Taenio Solium et Diphyllbothrium latum.

Malheureusement, les anticorps immunisants ou sensıbilısants sont généralement hétérologues dans le cas d'espèces, de genres et même de familles proches. Les réactions obtenues sont des réactions de groupe, peu spécifiques et d'interprétation assez délicate (BRISOU, 1946 ; DESCHIENS et POIRIER, 1952). En médecine vétérinaire, ce sont les réactions allergiques cutanées qui sont les plus pratiques et les plus simples (EUZÉBY, 1958). L'intra-dermo-réaction (I. D. R.) de Casoni a été employée dans le dépistage de l'échinococcose et de la cénurose du mouton, principalement en U. R.S.S.

En ce qui concerne la cysticercose bovine, les premiers travaux remontent à 1941 (SKVORTSOV, SOKOLOVA et TALIZIN). L'antıgène obtenu à partir des scolex du kyste hydatique donne 22 p. 100 de réactions positives sur les 72 bovins mis en expérience. Malheureusement, un seul animal porteur de 72 cysticerques fut autopsié par la suite, ce qui est insuffisant pour permettre de tirer des conclusions définitives.

En 1960, DEWHIRST, TRAUTMAN, PISTOR et REED arrivent aux mêmes conclusions à partır d'un antigène dont ils ne précısent pas la nature: sur 499 bovins soumis à l'l. D. R., 69 furent reconnus positifs et ce chiffre fut intégralement confirmé à l'autopsie.

En 1961, BUGYAKI constate, au Congo, que l'antigène total de Cysticercus bovis donne de bons résultats dans le diagnostic de la cysticercose bovine, mais que des réactions crolsées existent avec Fasciola hepatica et Dicrocolium dendriticum. 
En 1962, LEIKINA, MOSKVIN, ZORIKHINA et USTINOVITCH, expérimentent sur 11 veaux infestés artificiellement avec des oncosphères de Taenio saginata deuxtypes d'antigènes: le premier est fabriqué à partir du cysticerque complet et le second est un composé polysaccharide-protéine résultant du fractionnement de l'antigène total. En I. D. R., le premier s'est montré bequcoup plus spécifique que le second.

En 1963, FROYD reprend les expériences précédentes à KABETE au Kenya. Il travaille sur un grand nombre d'antigènes' entiers ou fractionnés provenant de Cysticercus bovis, Taenı saginata, Fasciola hepatica, fosciola gigantica, Echinococcus granulosus et Cysticercus fasciolaris. Le résultat de ses expériences indique qu'aucun de ces antigènes n'est spécıfique et que la méthode ne peut être valablement recommandée pour le diagnostic de la cysticercose bovine du vivant de l'animal.

Au Laboratore de Farcha (Fort-Lamy), deux séries d'essais ont été effectués :

10 Le premier, en 1962, avec l'antigène total de Cysticercus bovis qui sera appelé, pour plus de commodité «A. T. C. B.».

\section{a) Matériel et méthode.}

La technique est celle décrite classiquement pour la préparation des antigènes parasitaires.

Des Cysticercus bovis vivants ont été prélevés à l'abattoir, débarrassés du tissu réactıonnel environnant et finement broyés, en ajoutant $0.1 \mathrm{ml}$ d'eau distillée par parasite. Quand les cysticerques sont très nombreux, l'adjonction d'eau distillée n'est pas nécessaire!

L'émulsion est laissée $24 \mathrm{~h}$ à $+5^{\circ} \mathrm{C}$ dans le bas d'un frigidaire, en l'agitant de temps en temps. Le lendemain, elle est centrufugée et le lıquide surnageant sert d'antigène.

Le pli caudal a été choisi comme lieu d'élecfion. Deux injections intra-dermiques sont faites à droite et à gauche, l'une avec de l'eau distıllée stérile ef l'autre avec l'antigène $(0,1 \mathrm{ml}$ par animal).

La réaction allergique, sur un animal infesté, se traduit par un cedème chaud et violacé que l'on apprécie au pied à coulisse par comparaison avec le témoin et avec l'étaí du pli caudal avant l'intra-dermique. La plupart des auteurs (BUGYAKI, 1961, DEWHIRST, 1960, FROYD, 1963) considèrent que l'œdème doit atteindre une certaine taille, 4 à $5 \mathrm{~cm}$ et plus, pour que le test solt considéré comme positif.

Les réactions tardives étant d'ordre anaphylactique, la lecture se fait dans les 3-4 $h$ qui suivent l'injection.

56 animaux ont été ainsi soumis à l'l. D. R. et autopsiés quelques jours plus tard.

\section{b) Résultots.}

Le tableau $n^{\circ} \mathrm{XX}$ en donne l'essentiel :

TABLEAU $\mathrm{Y}: \mathrm{IX}$

I. D.R. - Antigène total Gysticercus boris

\begin{tabular}{|c|c|c|}
\hline Perasites & Réactiong positives & Réactions négatives \\
\hline Nématodes diverg & 0 & 5 \\
\hline Nématodes + Parenghi atomes & 0 & 1 \\
\hline Cestodes & 1 & 0 \\
\hline Shigtosomes & 3 & 4 \\
\hline Fasciala gigantica & 4 & 0 \\
\hline Fasciola gloantica + Shistosomes & 17 & 2 \\
\hline Fanciola + Shi stosomes + Cestodes & 3 & 0 \\
\hline Fasciola + Echinococcus + Shistasomes & 2 & 0 \\
\hline Cysticercus bovis & 5 & 1 \\
\hline Cysticercus bovis + Shistosones & 0 & , 2 \\
\hline Fasciola + Cysticercus bovis & 2 & 1 \\
\hline Fasciola + Cyaticercus bovis + Whistosones & 2 & 0 \\
\hline Fasclole + Cysticercus bovia + Cestodes & 1 & 0 \\
\hline
\end{tabular}


Les résultats figurant au tableau no IX coïncident donc exactement avec ceux de FROYD (1963) : même dans le cas de cysticercose pure, les animaux ne réagissent pas tous positivement et les réactions négatives sont le fait de porteurs de lésions anciennes calcifiées.

Des réactions croisées existent non seulement avec Fasciola hepatica, Fasciola gigantica, Dicroccelium dendriticum, Echinococcus polymorphus, mais encore avec des Cestodes comme Thysa, niezia ovilla et, une fols sur trols, avec certains Trématodes comme Shisfosoma bovis.

Nématodes et Paramphistomes donnent toujours des réactions négatives.

$2^{\circ}$ Deuxième essai : Antigène «Moniezia» (A. M.-Automne 1963).

Il existe une parenté assez étroite entre Moniezia expansa du mouton et Taenia saginata de l'homme (KENT et MACHEBCEUF, $1947 a$ et $b$; 1948). Plus récemment, LAMY, BENEX et GLEDEL (1959) démontrent par la déviation du complément que des réactions croisées sont possibles entre Taenia saginata et Moniezia expansa.

Il était donc intéressant de savoir si l'antigène préparé à partir de Moniezia expansa pouvaił être préconisé dans le diagnostic de la cysticercose bovine : Moniezia expansa est un Cestode cosmopolite, appartenant à la famille des Anoplocephalıdae, très fréquent chez,le mouton, la chèvre, le zébu et le chameau. Sa taille et son poids (jusqu'à $450 \mathrm{~g}$ ) en font potentiellement un bon producteur d'antıgène.

La technique de préparation est la même que celle décrite à propos de Cysticercus bovis. Seuls, les derniers, annecux ont été prélevés, car, d'après certains auteurs, les proglottis mûrs sont plus riches en antigène que ceux qui ne le sont pas. Le matériel ainsi constitué est lavé soigneusement 5 à 6 fois dans du sérum physiologıque et broyé sans adjonction de liquide. L'émulsion est placée dans un réfrigérateur pendant $24 \mathrm{~h}$, agitée régulièrement, puis centrifugée le lendemain à 4.000 tours. Le liquide surnageant, trop épais, est filtré sur Seitz et conservé à $+5^{\circ} \mathrm{C}$.

52 anımaux de toute provenance ont été choisis. L'inoculation intra-dermique de $0,1 \mathrm{ml}$ d'antigène «Moniezia» par animal a été faite dans le plı de la queue d'une part et dans le derme de l'encolure d'autre part. La lecture des résultats a lieu 3 à 4 h après l'inoculation et les animaux ont été sacrifiés 2 à 3 jours plus tard.

L'interprétation des réactions, selon la technique exposée plus haut, mène aux conclusions suivantes (Tableau $X$ ) :

I.D.R. - "Antirène Honiezia".

\begin{tabular}{|c|c|c|c|c|}
\hline & \multicolumn{2}{|c|}{ Pli caudal (11 animanx) } & \multicolumn{2}{|c|}{ Encolure ( 41 animaux ) } \\
\hline & $\begin{array}{l}\text { Réactions } \\
\text { positives }\end{array}$ & $\begin{array}{l}\text { Réactions } \\
\text { négatives }\end{array}$ & $\begin{array}{l}\text { Réactions } \\
\text { pogitives }\end{array}$ & $\begin{array}{l}\text { Réactions } \\
\text { négatives }\end{array}$ \\
\hline Cysticercus bovis & 1 & 0 & 3 & 2 \\
\hline c. bovis + Shistosomes & 1 & 1 & 3 & 6 \\
\hline C. bovis + Koniezia & 2 & 0 & & \\
\hline Montezia & & & 1 & $0^{\prime}$ \\
\hline Thysaniezia + Shistosomes & & & 1 & 0 \\
\hline Shigtosoma bovis & 2 & 1 & 6 & B. \\
\hline Fasciola + Shretosomes & & 2 & 1 & 3 \\
\hline Pas de parasites & - & 1 & 1 & -6 \\
\hline
\end{tabular}

Les réactions croisées avec Fasciola glgantica paraissent mans nambreuses que dans le cas précédent. Elles sont quasi constantes lorsque des Cestodes intestinaux (Moniezia ou Thysaniezia) sont présents. En outre, près de la moitié des animaux porteurs de Shistosomes donnent des réactions positives qui existent même avec des bovins totalement dépourvus de parasites.

Lorsque les cysticerques sont seuls, l'antigène «Moniezia, » ne permet de les déceler que dans 50-60 p. 100 des cas, contre 70-80 p. 100 avec 
l'A. T. C. B. Les réactions négatives concernent des cysticerques anciens et calcifiés.

\section{Conclusions.}

L'intra-dermo-réaction, en matière de cystıcercose bovine, ef quelle que soit l'origine de l'antigène employé (Toenı soginata, Echinococcus granulosus, Cysticercus fasciolaris, Cysticercus bovis, Fasciolo repotica, Fasciolo gigantica, Moniezia expansa), ne peut être valablement recommandé dans le diagnostic ante-mortem de l'affection.

Deux cas doivent être envisagés :

a) L'animal n'héberge que des cysticerques, d̀ l'exclusion de tout autre parasite. II ne faut pas compter dépasser 70-80 p. 100 de réactions positives avec I'A. T. C. B. et 50-60 p. 100 avec l'antigène «Moniezia».

b) Si les cysticerques sont associés d̀ d'autres helminthes de l'appareil digestif, de l'appareil circulatoire ou du foie, les résultats sont complètement faussés par les réactions de groupe et il est alors paradoxalement plus facile de déceler des Shistosomes, des Cestodes intestinaux ou des Distomes hépatiques que Cysticercus bovis. Comme les associations parasitaires sont très nombreuses dans un pays comme le Tchad, la méthode, pour l'instant, n'est guère susceptible d'applications pratıques.

\section{B. - Post-morfem}

La question a déjà fait l'objet de nombreux développements (VILJOEN, 1937 : GRABER, 1959 ; LEINATI, MARAZZA, GRIMALDI et PERSIANI 1963). II est un point sur lequel il importe cependant d'insister : celui de l'utılısation de la lumière de WOOD (U. V.) pour la mise en évidence des cysticerques présents dans les viandes. Si tel est le cas, une fluorescence roserouge se développe dont est responsable le liquide renfermé dans la vésicule.

Un certaın nombre d'auteurs se sont penchés sur ce problème (KOLLER, 1943; TOLGAY, 1953 ; BRANDES, 1958 ; LERCHE ef ELMOSSALAMI, 1959; SENS, 1960; MARAZZA et PERSIANI 1960, 1961; LEINATI, MARAZZA, GRIMALDI ef PERSIANI, 1963).

La méthode permet de mettre en évidence les cysticerques qui ont échappé à l'inspection telle qu'elle est pratiquée dans les abattoirs. LERCHE ef ELMOSSALAMI (1959), à la lumière de WOOD trouvent 1,8 p. 1.000 de nouveaux cas de Cysti- cercose dans 2.000 carcasses et 15,5 p. 1.000 dans les 385 têtes examınées. L'emploi des U. V. se justifie quand les Cysticerques sont vivants ou morts, mais non dégénérés. Le froid ne modifie pas les résultats. Si la viande parasitée est stérilisée à la chaleur, la fluorescence rose disparaît.

Il serait intéressant d'essayer cette méthode au Tchad.

\section{IV. - RÉSISTANCE DES ANIMAUX A L'INFESTATION. IMMUNITÉ}

\section{Essais d'infestation.}

a) Matériel ef méthode.

En février 1961, 22 bouvillons de 12 à 22 mois ont été achetés dans des élevages locaux de la région de Fort-Lamy, dans un milieu où le taeniasis humain touche environ 0,5 p. 100 de la population (GUILHON, GRABER ef GELLER, 1960 ; Service de Santé du Tchad, communication personnelle, 1964).

Les œufs de Taenia saginata ont été recueillis à partir de proglottis fournis par des manœuvres de l'abattoir de Fort-Lamy. Les segments ont été placés dans une solution saline de Merthıolate (Sérum physiologıque normal, Merthiolate au $1 / 10.000$ ) et conservés pendant $24-28 \mathrm{~h}$ à $+5^{\circ} \mathrm{C}$.

Juste avant l'emploi, les anneaux ont été grossièrement broyés dans un mortier śtérile et les œufs comptés. Les tests de viabilité ont été effectués selon la technique de SILVERMAN (1954 a), après incubation dans du liquide intestinal artificiellement reconstitué. Les doses infestantes onf été administrées par la voie buccale « d̀ la boutelle ».

Les animaux ont été autopsiés de une d̀ 21 semaines après l'infestation ef l'age des cysticerques apprécié conformément aux critères donnés par GIBSON (1959), Mc INTOSH et MILLER(1960), SILVERMAN et HULLAND (1961)(*).

\section{b) Résultais. (Tableau XI)}

Au total, sur 22 animaux :

3 n'ont pu être infestés.

10 présentaient des cystıcerques miorts caséeux ou en grande partie calcifiés.

2 hébergeaient à la fois des cysticerques vivants et des cysticerques morts.

(*) Les parasites, après récolte, ont été placés dans de la bıle de bœuf fraîche à $39^{\circ} \mathrm{C}$ et leur évagination dans le liquide indique s'ils sont vivanls ou morls. 
TABLEAU NO XT

Infestation expérimentale de bouvillons par des oeufs de Taema saginata.

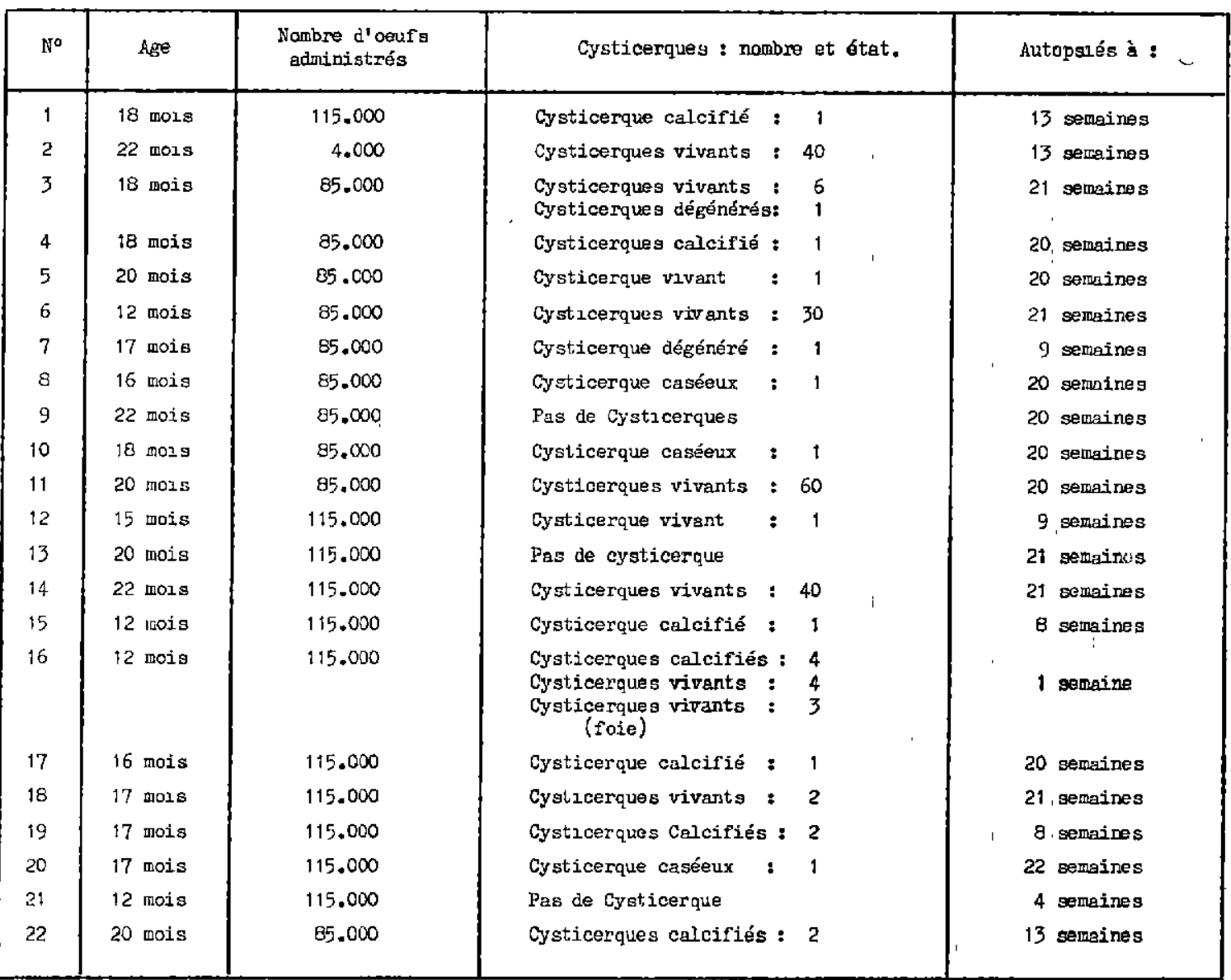

7 bouvillons ont développé des cysticerques vivants, bien caractéristiques après l'infestation.

Le nombre de cysticerques rencontrés est sans commune mesure avec le nombre d'œufs administrés :

85.000 œufs : 1 d̀ 40 cysticerques vivants, 115.000 œufs : de 2 à 40 cysticerques vivants.

Après une infestation expérimentale, les cas de cysticercose généralisée paraissent proportionnellement plus nombreux que lors d'infestation naturelle.

\section{c) Discussion.}

1) Dans les conditions du Tchad, la présence d'un seul cysticerque vivanł ou dégénéré, même calcifié, suffit à empêcher la réinfestation des anımauxatteints, lorsqu'ils absorbent des anneaux ou des œufs de Toenia saginata sur le sol ou dans l'eau. C'est ce qu'avaient déjà vu, en Australie et au Kenya, PENFOLD, PENFOLD ef PHILIPS (1936 $b ; 1937)$, FROYD $(1960 ; 1961)$ et URQUARTH (1961).

2) Cette règle n'a cependant pas une valeur absolve car, dans deux cas de l'expérience précédente, la coexistence simultanée de cysticerques vivants et de cysticerques morts a été observée.

On sait que, dans un certain nombre d'dffections (peste bovine notamment), les jeunes veaux ne sont pas de bons producteurs d'anticorps : SOULSBY (1963), en matière de cysticercose bovine, confirme également cette façon de voir : trois veaux de moins de trois mois, infestés artificiellement et suivis régulièrement, ont une mauvaise réponse sérologique ef le taux d'anti- 
corps décelables est extrêmement faible, ce qui n'est pas le cas sı les anımaux acquièrent la cysticercosevers l'âge de 4-6 mois.

Or, les veaux sont capables de contracter l'affection soit avant la nasssance (Mc MANUS 1960 et 1963), soit dans les premiers jours ou les toutes premières semaines de leur existence (URQUARTH, 1958 et 1961). SI l'on sult l'idée de SOULSBY (1963), les anticorps produits à ce moment, ne sont pas, dans un certain nombre de cas, 'en quantité suffisante pour neutraliser des infestations ultérieures. C'est certaınement là l'explication de la présence simultanée sur le mêrite animal de cysticerques morts et de cysticerques vivants, les premiers apporiant la preuve d'une contamination très précoce, pas toujours suivie d'ımmunité.

Au Tchad, il semble bien que l'infestation des veaux de lait de l'Ouest Tchadien ne soit pas tellement fréquente au départ (voir tableau no 1), d'où, par la suite, le petit nombre de' bouvililons porteurs de cysticerques morts et vivants. Que ce soit dans les conditions expérimentales ou naturelles, le taux ne dépasse pas 9.p. 100. Dans d'autres pays d'Afrique, Il est beaucoup plus élevé (dans les expériences d'URQUARTH au Kenya, il se situe autour de 25 p. 100).

3) Les infestations sont totalement négatives dans presque le $1 / 5$ des cas (17-19 p. 100). Là encore, URQUARTH (1961) a noté le même phénomène au Kenya. On peut admettre :

que des cysticerques ont échappé à l'examen, qu'il s'agit d'une résistance innée, naturelle, que l'immunité a été obtenue très tôt, à la faveur d'une infestation légère et que les cysticerques ont très rapidement disparu.

4) Au toial, l'expérience prouve que, vers l'âge

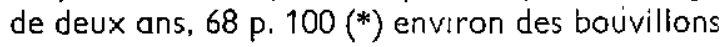
ne sont pas susceptibles d'être infestés artificiellement. Ils l'ont été ou le sont déjà naturellement :

soit qu'ils alent été en contact, dès les premiers jours de leur existence, avec des cufs de $T$. saginata : l'immunité provoquée est alors, dans l'ensemble de médıocre qualité.

(*) Ces chiffres sont bien supérieurs à ceux du tableau $n^{\circ} 2$. Il faut tenir compte, en plus des animaux naturellement résistants, des cysticerques oubliés à l'autopsie (d peu près $10 p .100$ ) dont la recherche a été faite de façon plus superficielle que lors des essais d'infestation expérimentale où les muscles el les organes ontété littéralement mis en pièces. sait que le contact ait eu lieu plus tard, vers 5-6 mois : l'immunité est alors solide ef les réinfestations ne paraissent guère possibles, tout au moins tant que le cysticerque est en place.

soit qu'ils fassent preuve d'une résistance naturelle, innée ou acquise, la mise en évidence des cysticerques se révèle impossible à l'autopsie (environ 17-19 p. 100).

5) II apparaît donc que 32 p. 100 environ (*) des bouvillons évitent l'infestation dans les deux premières années de leur existence : l'infestation est alors réalisable expérimentalement ou naturellement.

Cette opınion est confirmée indirectement par les statistiques de l'abattoir de Bangui sur, du bétali de boucherıe (6 à 10 ans) acheté auTchad et acheminé par la route depuss Fort-Archambault jusqu'à Bangui, dans des conditions qui ont été décrites plus haut.

Les chiffres donnés au Tableau no $\mathrm{VI}$ montrent que le pourcentage moyen d'animaux atteints. de cysticercose est de 29 p. 100 des, 45.000 têtes sacrifiées de 1954 à 1959.

Les deux chiffres ( 32 p. 100 et $29 \mid$ P. 100 ) sont donc très voisins. II ne s'agıt pas là d'une simple coïncidence : placés dans des conditions d'infestation naturelle très sévères, le tiers seulement des animaux de boucherie est susceptible d'être atteint de cysticercose. Ce sont, compte tenu' de ce qui sera dit plus loin à propos de la longévité du parasite, ceux qui ont échappé à l'immunisation dans les premiers mois de leur existence.

D'alleurs, ce qui est vrai pour l'abattoir de Bangui, l'est également pour certaines régions du Tchad (Mayo-Kebbi) ef du Nord-Cameroun (Maroua) : nulle part le taux d'infestation des zébus adultes ne dépasse 30 p. 100.

Un autre argument qui plaide, pro parte, en faveur de cette thèse est la forte proportion de cysticerques vivants rencontrés chez les vieux animaux parasités : 45 p. 100 dans le ChariBaguirmi, 82 p. 100 à Fort-Archambault, 77 p. 100 à Bongor ef 72 p. 100 à Maroua, ce qu semble prouver l'ınfestation récente d'animaux réceptifs, non immunisés antérieurement.

Cet état de choses, dans les zones sahéliennes. du Tchad, est imputable vraisemblablement aux

(*) Ce chiffre n'est valable que pour les années 1959 , $1960^{\circ}$ el 1961 , années couvrani la vie économique des. animaux mis en expérience. 
conditions climatiques et humaines propres à ces régions : la chaleur et la sécheresse détruisent, 5 mois de l'année durant, un grand nombre d'œufs de Taenia à la surface du sol ; les mares permanentes sont relativement rares et les porteurs humains de Cestodes ne représentent qu'une infime partie de la population (*) qui est ellemême très clairsemée.

Les conditions changent déjà plus au Sud, notamment au Mayo-Kebbi et dans la région de Maroug (Nord-Cameroun) : population dense; humidité plus élevée : exıstence de lacs, rivières ef mares importantes qui sont autant de sources d'infestation toute l'année.

6) Le problème du devenir des cysticerques a falt couler beaucoup d'encre. PENFOLD (1937) estime, à la suite d'expériences effectuées en Australie, que les cysticerques ne sont pas capables de survivre plus de neuf mois et qu'ils meurent, pour la plupart, vers le quatrième mois, ce que contestent JEPSEN et ROTH (1947). FROYD (1960), puis URQUARTH (1961) pensent que la survie peut être de plusieurs années. Mc INTOSH et MILLER (1960) avancent le chiffre de 55 semaines et DEWHIRST, CRAMER et PISTOR (1963) celui de 639 jours.

II est difficile de trancher la question. II est probable que, dans certains cas, quelques cysticerques peuvent demeurer vivants, donc dangereux, toute la vie économique de l'anımal. Ce n'est cependant pas une règle constante. La dégénérescence des cysticerques a été bien étudiée par SILVERMAN et HULLAND (1960). La réponse de l'hôte infesté est variable: il s'agit d'une réaction inflammatoire avec phagocytose non spécifique. Plus elle est importante, et moins le kyste parasitaire a de chance de se développer. C'est ce qui se passe dans les organes comme la langue, le cour et le foie où l'inflammation aigue devient chronique deux mois après l'infestation expérimentale, alors que ce stade n'est atteint que trois mols plus tard seulement dans les muscies où la réaction d'origine est moins violente. En outre, d̀ l'intérieur d'un même organe, i) existe parfois des différences dans l'évolution du processus inflammatoire.

(*) 0,5 p. 100 à Fort-Lamy; 2 à 3 p. 100 d̀ Abécher ; 1,1 p. 100 à Fort-Archambault (communication Service de Santé du Tchad, 1964).
II semble bien qu'intervienne, ò proximité des cellules qui entourent le kyste, une substance qui n'est pas un anticorps spécifique dont l'action se ferait sentır plutôt sur l'œuf de Taenı saginato au niveau de l'intestin de l'hôte intermédiaire (SILVERMAN, 1955 ; FROYD, 1960). II est évident, dans ces conditions, que la dégénérescence du cysticerque sero plus ou moins rapide : elle paraît être sous la dépendance de facteurs qui sont d'ordre strictement individuels, d'où les écarts observés d'un pays à l'autre et d'un animal à l'autre. PENFOLD (1937) en faisait déjà la remarque : «La longévité des cysticerques varie beaucoup chęz un même animal comme sur des animaux différents ». Récemment FROYD (1964 b) constate lui aussı que, si l'infestation a lieu très tôt, les cystıcerques sur le même animal meurent peu à peu à des degrés d'évolution différents et qu'un certain nombre d'entre eux sont capables de survivre bequcoup plus longtemps qu'il n'est classiquement prévu.

Au Tchad, la dégénérescence caséeuse, puis calcaire a lieu assez tôt : vers deux ans, 70 p. 100 des cysticerques rencontrés chez les bouvillons sont déjà presque entièrement calcifiés.

7) Le problème de la cystıcercose bovine, ces dernières années, a été étudié à fond au Kenya et des auteurs, comme FROYD et ROUND (1959, 1960) : URQUARTH (1958, 1959, 1961) ont porfaitement exposé les facteurs locaux qui président à la dissémination de la maladie. Ce sont, par rapport aux conditions tchadiennes:

chaleur dans l'ensemble beaucoup plus faible, degré hygrométrique plus élevé, d'où survie des œufs de Taenia saginato assurée pendant un laps de temps plus long.

Taux de taeniasis humain volsin de 30 p. 100 (FENDALL, 1959) contre 0,5-3 p. 100 au Tchad.

Infestation quasi constante des veaux, soit prénatale, soit dans les tout premiers jours de leur existence.

Présence simultanée de cysticerques vivants et de cysticerques morts plus fréquent qu'au Tchad.

Abattage du bétail de boucherie plus précoce ( 5 ans, contre 7 à 11 ans en moyenne au Tchad).

Différences tenant au mode d'élevage (plutôt de type sédentaire) au Kenya et à la présence d'animaux destınés à la production de lait.

Longévité apparemment plus grande des cysticerques dans les tissus de l'hôte. 
Taux d'infestation des adultes plus élevé qu'au Tchad.

Peuplement humain plus abondant.

Consommation de la viande de bœuf plus fréquente au Tchad chez les sédentaires, que chez les nomades.

On peut se demander, s'il ne faudrait pas envisager à l'échelon du continent africain, deux types de cysticercose bovine, le premier valable pour les pays secs et chauds, à faible densité humaıne et ou le taeniasis est peu répandu, le second pour des pays plus humides, plus peuplés et où l'incidence du taeniasis humain est importante.

\section{V. - TRAITEMENT}

Il y a déjà quelques années, des essais de traitement ont été effectués avec des substances telles que l'huile thymolée à 50 p. 100 (HERIN et THIENPONT, 1957) et l'acide oxalique (GINSBERG, 1958 ), sans aucun succès d'ailleurs.

Plus récemment, URQUARTH (1960) utilıse le Diethylcarbamazine (Notezine) à des doses de $10-40 \mathrm{mg} / \mathrm{kg}$ administrées pendant 7 à 10 jours. Les résultats ont été décevants et la plupart des cysticerques sont encore vivants après le traitement. En Uganda, ROLLINSON (1960) avec des composés phosphorés orgoniques comme «le Ruèlène ou l'Etrolène "n'obtıent pas une diminution significative du nombre de cysticerques deux mois après le traitement.

Au Laboratoire de Farcha, ont été expérimentés deux anthelminthiques nouveaux :

le 2 (4'-Thiazolyl) Benzimidazole, plus connu sous le nom de Thiabendazole Merck.

le 2,2'-Thiobis (4,6-Dichlorophenol) ou Actamer ou Bithionol.

a) Le Thiabendazole a été recammandé dans le traitement de la trichinose musculaire du porc (CAMPBELL ef CUCKLER, 1962), à la dose de 0,3 p. 100 dans la ration pendant sept jours.

En ce qui concerne la cysticercose bovine, les essais ont été portés sur une dizaine d'anımaux naturellement infestés. Des doses uniques allant de 50 à $750 \mathrm{mg} / \mathrm{kg}$ ont été distrıbuées. Une semaine après, à l'autopsie, les cysticerques recueillis s'évaginent normalement dans la bıle de bœuf à $+39^{\circ} \mathrm{C}$, ce qui indique bien qu'ils n'ont pas été touchés par le médicament.

b) L'Actamer, sur une quinzaine d'anımaux, ne donne pas de mellleurs résultats, quelle que soit la dose emplayée (de 10 à $45 \mathrm{mg} / \mathrm{kg}$ ).

\section{VI. - ESSAIS D'IMMUNISATION}

Tout récemment, divers auteurs se sonł penchés sur le problème de l'immunisation des jeunes anımaux.

URQUARTH et Coll. (1963) admınıstrent à des veaux des œufs de Taenia saginata irradiés à $40 \mathrm{kr}$. Ces animaux soumıs de 34 à 42 jours plus tard à une infestation massive à partır d'œufs non ırradiés de Toenio saginata montrent ò l'autopsie sept à vingt fols moins de cysticerques que les animaux témoins.

Cependant la méthode n'est pas très pratique, car Il Importe d'une part de se procurer chez l'homme une quantıté suffisante de matériel Infestant et d'autre part de « vacciner » les veaux avant qu'ils n'entrent en contact avec des excréments humains, ce qui est difficile dans les conditions africaines.

De son côté, FROYD (1964 a) injecte à des veaux divers sérums prélevés sur des veaux hautement infestés par Cysticercus Bovis. Les animaux ainsi immunisés reçoivent des quantités considérables d'cuifs de Taenio saginata. Les résultats n'ont pas été favorables et ll'ımmunité passive contre Cysticercus bovis ne peut être conférée à des veaux, même si les dosès de sérum sont très élevées.

\section{CONCLUSIONS}

1. - En République du Tchad, la cysticercose bovine frappe environ 2 p. 100 des veaux de lait abattus.

Le taux d'infestation des bouvillons a considérablement augmenté passant de 16 p. 100 (1957) à plus de 32 p. 100 (1961-1963). 8 à 12 p. 100 des adultes sont porteurs de vésicules, sauf au MayoKebbi où le pourcentage attent 22 p. 100.

2. - Les sources d'infestations sont constituées par les anneaux de Toenia saginata expulsés, les œufs présents dans les selles des individus atteints et par les «œufs anaux» dont le rôle paraît essentiel dans l'infestation des veaux.

3. - Les œufs semblent soumis, pendant 5 mass de l'année, à des conditıons extérieures 
très dures dont les deux principales sont la sécheresse de l'air et la chaleur au sol. Ces facteurs climatiques limitent sans doute la résistance des ceufs dans le temps ef diminuent ainsi les chancés d'infestation du bétail.

Les auteurs insistent particulièrement sur l'importance des collections d'eau permanentes riches en oufs de Taenia saginata, dans la transmission de la cysticercose aux bêtes de boucherie qui se rendent à pied dans les territorres vossıns (R. C. A.) où elles seront abattues.

4. - Les variations saisonnières dans la fréquence de la cysticercose bovine n'obéissent à aucune règle particulı̀re : elles varient selon les régions et l'âge des animaux.

5. - Le diagnostic ante-mortem de la cysticercose est aléatoire. Les nombreux antigènes employés en intradermo-réaction donnent des réactions de groupe avec les Cestodes intestinaux et divers Trématodes. Cette méthodo est donc inutilisable dans un pays comme le Tchad où le polyparasitisme est de règle.

6. - L'immunité conférée par une première contamination paraît solide et semble-t-il du rable, à condition que l'infestation initiale se place assez tard, c'est-à-dire vers le $4 \mathrm{e}$ ou le $5 \mathrm{e}$ mors de la vie de l'animal.

Si l'infestation est trop précoce (de quelques jours à trois mois), la production d'anticorps est insuffisante pour neutraliser l'embryon hexacanthe de Taenia saginata. Des surinfestations ou des réinfestations risquent de se faıre jour, avec présence simultanée, sur un même animal, de cysticerques vivants et de cysticerques morts :

Un certain nombre d'animaux jouissent d'une immunité naturelle, peut-être innée, le problème r'est pas encore complètement élucidé.
Les essals d'infestation effectués au Laboratorre de Farcha ont, en outre, démontré qu'avant l'âge de deux ans, les deux tiers des bouvillons sont déjà parasités. Le dernier tiers échappe à l'infection et n'est donc pas immunisé. Cette façon de vorr est confirmée par les chiffres relevés, duranf quelques années, à l'abattoir de Bangui (R. C. A.) sur des bovins adultes venus à pied des zones du Tchad (29 p. 100 en moyenne de saisie pour cysticercose) et par le fait que le taux d'infestation des zones très touchées (NordCameroun, Mayo-Kebbı) ne dépasse pas 30 p. 100.

7. - Les différences constatées entre le Tchad, pays sec et chaud, faiblement peuplé, où le taux de téniasis humain est faible $(0,5-3$ p. 100) et le Kenya par exemple, pays beaucoup plus humide et ou $30 \mathrm{p} .100$ des individus sont porteurs de Taenio saginato, laissent su'pposer l'existence en Afrique de deux types de cysticercose bovine dépendant étroltement des facteurs humains et climatıques locaux.

8. - Le traitement de la cysticercose avec des Anthelmınthıques modernes comme les composés phosphorés organiques; le Thiabendazole, l'Actamer ou la Notézine s'est constamment soldé par des échecs.

\section{REMERCIEMENTS}

Nous remercions MM. les Directeurs TROUETTE ef LAURENT, respectivement Directeurs de l'abattoir de Fort-Lamy et de l'abattoir de Bangui, d'avair eu l'amabilité de nous communiquer leurs statistiques et $M$. le Directeur du service de Santé du Tchad d'avoir bien voulu nous donner tous les renseignements concernant le téniasıs humain.

\title{
SUMMARY
}

\author{
Bovine cysticercosis in the republic of Chad \\ Comments on the present situation, the actiology, diagnosis, \\ immunity and treatment of this zoonose
}

\begin{abstract}
$1^{0}$ In the Republic of Chad, Bovine Cysticercosis, affects about 2 per 100 of veal calves. The rate of infection of young beef animals has increased considerably from 16 per 100 (1957) to 32 per 100 (1962-63), 8-12 per 100 of odult, cattle are infecled except at Mayo-Kebbi, where the percentage has reached 22 per 100.
\end{abstract}


$2^{0}$ The sources of infection are the eliminated segments of Toenio soginato, the eggs present in the stools of infected individuals and the «anal eggs», whose role is essential in the infection of calves.

3o During five months of the year, the eggs are submitled to very difficult climatical conditions, the main ones being the dryness of the alr and the heat of the ground. These factors certainly limit the period of viability of the eggs thus decreasing the chances of infection of cartile.

The authors would emphasize particularly the importance of slagnantwater, rich in eggs of $T$. soginata in the transmıssion of Cysicercosis to beef cattle which are driven to adjacent countries (R. C. A.) to be slaughtered.

$4^{\circ}$ The occurence of bovine Cysticercosis appears to have no marked seaso$\mathrm{nal}$ varialion. It varies according to the region and age of anımal.

$5^{0}$ The ante mortem diagnosis of Cysticercosis is uncertain. The numerous antigens employed in intra-dermal tests give group reactions with intestinal Cestodes and various Trematodes. This method is thus useless in a country such as Chad where polyparasitism is the rule.

$6^{\circ}$ The immunity conferred by an infection appears to be solid and it would seem, lasting, provided that the intial infection occurs fairly late, that is towards the 4 th or 5 th month of the life of the animal.

If the infection occurs too early (during the first few days of life up to the 3 rd month, the production of antibody is not sufficient to neutralize the hexacanth embryo of $T$. saginato. Further infections are likely to occur so that one may find concurrently in the same animal live and dead Cysticerci.

A certain number of animals possess a natural immunity, perhaps innate; this question has not yet been completely resolved.

Experiments carried out at the laboratory of Farcha, have shown that before the age of two years, two thirds of the steers are already infected with Cysticercus bovis. The remoining third escapes infection and thus receives no immunity. This information is confirmed by data accumulated over' several years at the slaughter-house of Bangui (R. C. A.) from caltle which have come on foot from regions in the north of Chad (32 per 100 were sized on account of Cysticercosis) and by the fact that the rate of infestation in the most infected zones and subject to similar climatic conditions (North Cameroun, Mayo-Kebbı), does not exceed 30 per 100 .

in certain cases, Cysticerci can prabably survive several years in the muscle of their hosis.

The differences between Chad, a country dry and hot, where the rate of human infection by Toenia is low, and Kenya, for example, a country much more humid, where 30 per 100 of the population carry T. saginato, lead one to suppose that there exists in Africa, two rypes of Bovine Cysticercosis depending closely upon human and local climatic conditions.

One map, 11 tables and 109 references accompany this paper.

\section{RESUMEN}

La Cisticércosis bovina en la Republica de Tchad. Algunas reflexiones en la presente situación, la etiologia, el diagnóstico, la inmunidad y el tratamiento de esta zoonosis

10 En la Republica del Tchad, la cisticércosis bovına se encuentra en cası 2 por 100 de los terneros de leche sacrificados.

Ha aumentado considerablemente el termino medio de infección de los novilJas, de 16 por 100, en 1957, a más de 32 por 100, en 1961-63.

8 a 12 por 100 de los adultos tienen vesiculas, excepto en el Mayo-Kebbi dónde el percentaje es de 22 por 100. 
$2^{\circ}$ Consilituyen las causas de infección los anillos del Taenía saginata expelidos, los huevos presentes en las heces de los individuos alacados y los « huevos anales » cuyo papel es esencial en la infección de los terneros.

$3^{0}$ Los huevos parecen sometidos, durante 5 meses del ano, a condiciones exteriores muy dificiles, entre las cuales la sequedad del aire'y el calor del suelo son las dos principales.

Estos factores climáticos limitan seguramente la resistencia de los huevos en el liempo y asi dismenuyen las probabilidades de infección del ganado.

Los autores insisten particularmente en la importancia de las reservas de agua permanente, ricas de huevos del T. saginata, en la propagación de la cisticércosis a los animales de carne que transhuman a los territorios vecinos (R. C. A.) dónde seran sacrificados.

40 Las variaciones según la estación en la frecuencia de la cisticércosis bovina no siguen ningün principio particular : varian con las regiones y la edad de los animales.

$5^{\circ}$ Es aleatorio el diagnóstico ante-mortem de la Cisticércosis. Los númerosos antigenos empleados en la intradermoreacción dan reacciones de grupo en los céstodos intestinales y diferentes tremátodos. Pués no se puede utilizar este método en un pais tal como el Tchad donde existe el poliparasitismo.

$6^{\circ}$ La inmunidad conferida por una primera contaminación parece solida y durable, con tal de que la infección inicial se situe demasiado tarde, es decir a eso del cuarto o quinto mes en la vida del anımal.

Si la infección es demasiado precoz (de algunos dias a tres meses), no es suficiente la producción de anticuerpos para neutralizar el embrión hexacanta del $T$. saginato. Arriesgan de aparecer superinfecciones o reinfecciones, con la presencia simultânea, en el mismo animal, de cisticercos vivos y de cisticercos muertos.

Un cierto número de animales gozan de una inmunidad natural, posiblemente innata, no se ha resuelto el problema completamente.

Ensayos de infección efectuados en el Laboratorio Farcha, han demostrado que antes de los dos años de edad, los dos tercios de los novillos han hecho ya o estan haciendo una Cisticércosis.

\section{BIBLIOGRAPHIE}

1. ANDRONIKASHVILI (R. V.) (1960). - The examination of carcasses infected with Cysticerciasis. Veterinarya, Moscou, 37,(9), 80-1

2. AVAKYAN (D. M.) (1961). - Teniasis in the Kafansk area. Medit. Parazitol. Parazitarn. Bol., 30, (2), 148-50.

3. BIAGI (F.) et TAY (J.) (1958). - A precipitation reaction for the diagnosis of Cysticercosis. Am. J. Trop. Med. Hyg., 7, (1), 63-5.

4. BICHE (Y.) ef THIENPONT (D.) (1959). - Etude statistique de la Cysticercose bovine au Ruanda-Urundi. Ann. Med. Vet., 103, (1), 27-35.
5. BIRKETT (J. D.) (1953). - Cysticercus bovis in the N'Dama cattle of Sierra-Leone. Vet. Rec., 65, (24), 391-4.

6. BRANDES (H.) (1958). - Untersuchungen zur Feststellung der Finnigkeit beim Rind unter Besonderer Berüksichtingung der Untersuchung mit filtrierten U. V. Strahlen. Arch. F. Lebensmittelhyg., 9, 241-3.

7. BRISOU (J.) (1946). - Diagnostic du kyste hydatique par extrait de Ténia. Bull. Soc. Poth. Exot., 39, (5/6), 193-6.

8. BUGYAKI (L.) (1961). - Diagnostic de la 
Cysticercose à l'aide de l'intra-dermo-réaction. Bull. Epiz. Dis. Afr., 9, 15-23.

9. CAMPBELL (W. C.) (1963). - The efficacy of surface-active agents in stimuling the evagination of Cysticerci in vitro. J. Parasit., 49, (1), 81-4.

10. CAMPBELL (W. C.) et RICHARDSON (T.) (1960). - Stimulation of Cysticercus evagination by means of surfactants. J. Parosit., 46, (4), 490.

11. CAMPBELL (W. C.) ef CUCKLER (A. C.) (1962). - Thiabendazole treatment of the invasive phase of experimental trichinosis in swine. Ann. Trop. Med. Parasit., 56, (4), 500-5.

12. CAMPBELL (W. C.) et CUCKLER (A. C.) (1962). - Effect of Thiabendazole upon experimental trichinosis in swine. Proc. Soc. Exp. Biol. Med., Il0, (1), 124-8.

13. CHANDLER (A. C.) (1956). - Introduction to Parasitology. New York, 350-7.

14. CULBERSTON (J. T.) (1941). - Immunity against animal parasites. New York.

15. DEOUELL (J.) (1957). - Problems of Cyst. bovls in general and in frozen meat plants in Abyssinia and Eritrea in particular. Ref. Vet., 14, (2), 57-63.

16. DESCHIENS (R.) et POIRIER (M.) (1952). L'immunité dans les infestations parasitaires. Ann. Inst. Post., 83, (6), 725-44.

17. DESPRES (P.) (1962). - Note complémentaire au problème de la Cysticercose bovine en Suisse. Schweiz. Arch. F. Tierheılk., 104, (2), 116-9.

18. DESPRES (P.) et RUOSCH (W.) (1961). Diagnostic et importance de la Cysticercose bovine en Suisse. Schwertz. Arch. $f$. Tierheilk., 103, (10), 507-18.

19. DEWHIRST (L. W.), TRAUTMAN (R. J.), PISTOR (W. J.) et REED (R. E.) (1960). Studies on ante mortem diagnostic procedure in bovine Cysticercosis Infections. J. Parasit. 46, (5) (Sect. 2), 10-11.

20. DEWHIRST (L. W.), CRAMER (J. D.) ef PISTOR (W. J.) (1963). - Bovine cysticercosis I.' Longevity of Cysticerci of T. saginata. J. Porasit., 49, (2), 297-300.
21. DOBY (J. M.), DOBY-DUBOIS (M.) et DEBLOCK (S.) (1957). - Fréquence de la Téniase par $T$. saginata chez 23.000 enfants de la région de Yaoundé (Cameroun), détectée par la méthode de Graham. Bull. Soc. Path. Exot., 50, (6), 929-36.

22. DUTHIE (B. L.) et VAN SOMEREN (V. D.) (1948). - The survival of T. saginata eggs. in open pasture. E. Afr. agric. J., 13, 149-152.

23. EISA (A. M.), MUSTAFA (A. A.) et 5OLIMAN (K. N.) (1962). - Preliminary report on Cysticercosis and hydatidosis in Southern Sudan. Sudan J. Vet. Sci. Anim. Husb., 3, 97108.

24. EL AFIFI (A.), FARMY (M. A. M.) et ELMOSSALAMI (E.) (1961). - A study on the morphology and distribution of $C$. bovis in the intermediate host with special reference to a peculiar case of Sudanese calf. Vet. Med. J. Gizo, 7, (7/8), 307-15.

25. ERSHOV (V. S.) (1956). - Parasitology and parasitic diseases of Livestock. Moscou, 99-107.

26. EUZEBY (J.) (1958). - Diagnostic expérimental des Helminthozes animales. Paris, 330-45.

27. FAIN (A.) et DE RAMMEE (O.) (1949), - Les Helminthes parasites de bovidés à Astrida (Ruanda-Urundi). Ann. Porasit. Hum. comp., 24, (3/4), 207-10.

28. FENDALL (N. R. E.) (1959). - Taeniasis of man. Coll. Helm. Anim. Dom. I. A. C. E. D/ C. C. T. A. Nairobi, 49, 26-39.

29. FUENTES (P. B.), NEGRETE (M. J.) et VILLABOLOS (P.R.) (1960). - Algunos factores fisicos y quimicos qui adectan la evaginaction d. C. cellulosae. Rev. Inst. Solub. E. enferm. Mexico, 20, (2), 103-28.

30. FRIEDRICH (J.) (1961). - Ein Beitrag zum Problem der Rinderfinne. Schl. U. ViehhofZeit., 6I, (1), 1-9.

31. FROYD (G.) et ROUND (M. C.) (1959). Infection of cattle with $C$. bovis by the injection of oncospheres. Noture, 184, (4697), 1510.

32.' FROYD (G.) (1960). - Cysticercosis and hydatid disease of cattle in Kenya. J. parasit., 46, (4), 491-6. 
33. FROYD (G.) et ROUND (M. C.) (1960). The artificial infection of adult cattle with C. bovis. Res. Vet. Scl., I, (3), 275-82.

34. FROYD (G.) (1961). - The artificial infection of calves with oncospheres of T. saginata. Res. Vet. Sci., 2, (3), 243-7.

35. FROYD (G.) (1963), - Intradermal tests in the diagnosis of bovine Cysticercosis. Bull. epiz. Dis, Afr., II, (3), 303-6.

36. GIBSON (T. E.) (1959), - The identification of Cysticercus bovis with special reference to degenerate Cysticerci. Ann. Trop. Med. Parasit., 53, (1), 25-6.

37. GINSBERG (A.), CAMERON (A.), GODDARD (W. B.) et GRIEVE (J. M.) (1956). Bovine Cysticercosis with particular reference to East africa. Bull. Epiz. Dis. Afr., 4, (1/2), 24-36.

38. GINSBERG (A.) (1958). - Helminthic zoonoses in meat inspection. Bull. Epiz. Dis. Afr., 6, (2), 141-9.

39. GINSBERG (A.) (1959). - Problems affecting meat hygien in under developed countries. Int. Vet. Cong. Madrid, 11, 757-9.

40. GINSBERG (A.) et GRIEVE (I. M.) (1959). Two unusual cases of liver Cysticercosis. Vet. rec., 7I, (30), 618.

41. GINSBERG (A.) (1960). - The detection of C. bovis in the abattoir. Vet. Rec., 72, (16), 310.

42. GRABER (M.) (1959). - La Cysticercose bovine. Son importance dans les zones sahéliennes d'Elevage de la République du Tchad. Rev. Elev. Med. Vet. Pays Trop., 12. (2), 121-143.

43. GRUJIC (I.) (1960). - Humana tenijaza i cisticerkoza svinja i goveda. Vet., Sarojevo, 9. (1), 109-117.

44. GUILHON (J.), GRABER (M.) ef GELLER (A.) (1960). - Essais de traitement du Téniasis humain par le 5,5' - Dichloro 2,2' Dihydroxydiphenylmethane. Bull. Soc. Path. Exot., 53, (4), 697-703.

45. HERIN (V.) et THIENPONT (D.) (1957). Sur un essai de traitement de la ladrerie bovine ef porcine par l'huile thymolée en injection intramusculaire. Ann. Med. Vet., 101, 141-6.
46. HERMUS (G.) (1961). - Die Auswirkung der Massnahmen zur Bekámpfung der Rinderfinne im Bezirk Leipzig. Monats. $f$. Veterinór., 24, 935-37.

47. HOFSTRA (K.) (1959). - Cysticercose en vleeskeuring. II. Tijds. V. Diergeneesk., 84, (10), 538-47, 349.

48. HOLZ (J.) ef PEZENBURG (E.) (1957). Histologie und histochemische Untersuchungen an den Hüllen von C. inermis. Monats. f. Tierhei, 9, 3.7-43.

49. JADIN (J.) et GIROUD (P.) (1959). - Présence de néo-rickettsies dans les tissus larvaires de $C$. bovis au Kivu et au RuandaUrundi. Bull. Soc. Path. Exot., 52, (4), 420-22.

50. JEPSEN (A.) et ROTH (H.) (1949). - Epizootiology of Cysticercus bovis. Resistance of eggs of Tdenia saginata. Rep. Int. Vet. Cong. (XIVth). London 2, 43-50.

51. KENT (N.) etMACHEBCEU (M.) (1947a).-Sur l'existence de cénapses protéines-acides biliaires chez les Cestodes. C. R. Acod. SCl. CCXXV, 539-40.

52. KENT (N.) et MACHEBCUF (M.) (1947 b). - L'existence de cénapses glycogéno-protéiques de Monieza expansa. $C . R$. Acad. Sci., CCXXY, 602-4.

53. KEULEN (A. VAN) (1959 a), - Epidemiology of Cysticercus bovis. Int. Vet. Cong. (16th), Madrid, 11, 753-4.

54. KEULEN (A. VAN) (1959 b). - Cysticercose en vleeskeuring. Tidj. $\vee$. Diergeneesk., 84, (10), 526-36.

55. KOLLER (R.) (1943). - Die Fuoreszenz einiger Parasiten im Fleish. Zeits. f. Fleish. u. Milching. 53, (19), 185-6.

56. KOUDELA (K.) (1959). — Vysledky jednoleteho pruzkumu uhrivosti skotu. Sborn. Ceskos. Akadzemédel, Ved. Vet, Med., 32, (6), 441-54.

57. $\operatorname{LAMY}\left(L_{1}\right), \operatorname{BENEX}(J$.$) et GLEDEL (J.) (1959).$ - Etude de la réaction de fixation du complémeni à divers antigènes de Cestodes chez le mouton. Deuxième note. Bull. Soc. Path. Exot., 52, (2), 193-8.

58. LAPAGE (G.) (1956), - Veterinary parasitology. Edinburgh, 342-8. 
59. LAZZARO (D. A.) (1961). - Cysticercosis bovina. Gac, Vet. Buenos-Aires, 23, (129, 1921.

60. LEINATI (L.), MARAZZA (V.), GRIMALDI (E.) ef PERSIANI (G.) (1963), - Le elmintiasi dell'uomo da alimenti dl origine animale. Clin. Vet., 86, 173-217, 242-257, 356404.

61. LEIKINA (E. S.) MOSKVIN (Z. N.), ZORIKHINA (V. I.) et USTINOVITCH (M. A.) (1962). - Methods for diagnosing bovine Cysticercosis in the living animal. Proc. Conf. All. Union Soc. Helm. Moscou, 111. 98-9.

62. LEE (H. H. K.), JONES (A. W.) et WYANT (K. D.) (1959), - Development of the taeniid embryophore. Trans. Am. Micros. Soc., 78, (4.) 355-7.

63. LERCHE (M.) et ELMOSSALAMI (E. S.) (1959). - Nachweis von Rinderfinnen im filtrierten U. V. - Licht. Berl. u. Münch. Tierärtz. Wochensh., 71, (7), 131-4.

64. LUCKER (J. T.) (1960). - A test of the resistance of $T$. saginata eggs to freezing. J. Porosit., 46, (3), 304.

65. LUCKER (J. T.) et DOUVRES (F. W.) (1960). - Survival of $T$. saginata eggs on stored hay, Proc. Helm. Soc. Whosh., 27, (1), 110-11.

66. MAMEDOV (A. A.) (1958). - Cysticerciasis in zebu cattle. Vet. Moscou, 35, (5), 73-4.

67. MARAZZA (V.) et PERSIANI (G.) (1960). Indagine cisticercoscopica in luce di Wood su carni bovine nazionali ed estere ammesse al libero consumo. At. D. Soc. It. Sci. Vet., 14, 381-3.

68. MARAZZA (V.) ef PERSIANI (G.) (1961). Indagine cisticercoscopica in luce di Wood su carni bovine nazionali ed estere ammesse al libero consumo. Arch. Vet. It., 12, (3), 202-26.

69. MARSBOOM (R.), PARYS (O. VAN) et BRODSKY (M.) (1960). - Contribution d̀ l'étude des localisations préférentielles des Cysticerques chez le gros bétail en Urundi. Ann. Med. Vet., 104, (4), 191-6.

70. MARZULLO (F.), SQUADRINI (F.) et TAPARELLI (F.) (1957). - Studio istochimico sui parassiti patogeni per l'vomo. Nota III : hana, T. saginata. Boll. D. Soc. Med. Chirur. Modeno, 57. (4), 327-31.

71. MASELLIS (G.DE) (1960). - Indagini epizoologiche sulla Cisticercosi bovina in Campanie. At. d. Soc. It. Sci. Vet., 14, 385-8.

72. MAZOTTI (L.) (1944). - Observaciones en 10 individuos parasitados con $T$. saginata Presencia de huevecillos en la region perianal $y$ en otras regiones cutanéas. Rev. Inst. Solub. y. Enfer. Trop., 5, 207.

73. MC INTOSH (A.) (1956). - Early stages of the larvae (C. bovis) of $T$. saginata. J. porosit., 42, sect. (2), 41.

74. MC INTOSH (A.) et MILLER (D.) (1960), Bovine Cysticercosis, with spécial reference to the early developmental stages of T. saginata. Am. J. Vet. Res., 21, (81), 169-177.

75. MC MANUS (D.) (1960). - Prenatal infection of calves with $C$. bovis. Vet. Rec., 72 , (41), 847-8.

76. MERKUSHEV (A. V.), ILIN (M. M.), KOTOVA (O. M.) et NIKULSHINA (O. A.) (1962). - Incidence of Cysticerciasis and Hydratidosis in animals slaughtered in the Voronezh region (In Russe) - Zapik. Voronezhs. Selkokhozyaits. Inst., 17, (2), 101-4.

77. MIJATOVIC (I.) (1961). - Ikricavost teladi. Vet. sarojevo, 10, (3/4), 509-11.

78. MÖNNIG (H. O.) (1956). - Veterinary helminthology and Parasitology-London.

79. NEČEV (T.) et AČKOV (M.) (1960), - Bobicavost goveda svinja zaklanih u skopskoj Klanici u periodu I. I. 49 do 31.12.58. Vet. Glasn. Belgrade, 14, (9), 693, 8.

80. NENADIC (M. B.) (1957). - Cysticerkoza govedi zaklanih na pljevaljskoj klaonici. Vet. Glasn. Belgrade, 11, (12), 1194-7.

81. NENADIC (B. M.) (1960). - Rezultati trogodisnjeg istrazivanja bobicavoste goveda aklanih na gradskoj klaonici u pljevljima. Vet. Sorajevo, 9, (3), 597-602.

82. NEVENIC (V.), MEKULI (E.) et RADOVIC (D.) (1960). - Bobicabost goveda s. podrucja peci okoline moguenost sirenja vec pregledanim mesom. Vet. Glosn., Belgrade, 14, (3), 197-198. 
83. PARLIER ( $\left.E_{1}\right)$ (1938), - La ladrerie bovine à Dakar. Rev. Med. Vef., 90, (2), 504-15.

84. PAWEL (O.) et JANICEK (J.) (1963 a). Možnosił upravy urhiveho masa ionizujicim zařenim. Vet. Med. Prague, 36, (2), $11-20$.

85. PAWEL (O.) et JANICEK (J.) (1963 b) Prodouženi udržnoste hoveziho a veproveho masa pri pouzjte devitalzacnich davek ionizy ujiciho zareni $k$ inaktivaci urhuv. Vet. Med., Prague, 36, (2), 121-30.

86. PEEL (C.) (1961), - - The influence of the age factor on $C$. bovis infestation in West Africa N'Dama cattle. J. Trop. Med. Hyg., 64, (9.) 239-42.

87. PELLEGRINI (N.) et BONO (G. DEL) (1957). - Rilievi e considerazioni sulla infestione de C. bovis in provincia di Pisa. At. D. Soc. H. Sci. Vet., 11, 726-30.

88. PELLEGRINI (D.) (1958). - La profillassi della Cisticercosi bovina. Vet. It., 9, (1), 25-38.

89. PENFOLD (H. B.) (1936). - The treatment of patients infested with $T$. saginata, with special reference to certain usual results. Med. J. Austr., 1, (12), 385-98.

90. PENFOLD (H. B.) (1937). - The life history of Cysticercus bovis in the tissues of the ox. Med. J. Austr., Suppl. I., 579-583.

91. PENFOLD (W.J.) etPENFOLD (H. B.) (1937). - $C$. bovis and its prevention. J. Helm., $15,37-40$.

92. PENFOLD (W. J.), PENFOLD (H. B.) et PHILIPS (M.) (1936 a). - A survey of the incidence of $T$. saginata infestation in the population of the state Victoria from January 1934 to July 1935. Med. J. Austr., I, (9), 82-285.

93. PENFOLD (W. J.), PENFOLD (H. B.) et PHILIPS (M.) (1936 b). - Axquired active immunity in the ox to $C$. bovis. Med. J. Austr., I, (13), 417-23.

94. PENFOLD (W. 3.), PENFOLD (H. B.) et PHILIPS (M.) (1937). - Taenia saginata, its growth and propagation. J. Helm., 15, 41-8.

95. PODYAPOLSKAYA (V. P.) (1960). - The eradication of Teniasis in U. R. S. S. Sovetsk. Med., 24, (5), 12-17.
96. RAILLIET (A.) (1893). - Traifé de zoologie médicale ef vétérinaire. Paris.

97. RAPIC (S.), BAIC (S.), JEMRIC (K.) et MAL$\mathrm{CIC}$ (B.) (1959). - Die rontgendiagnostick der schweinefinnigkeif. Berl. u. Muñch. Tierárzł. Wochensch. 72, (15), 300-3.

98. Rapports annuels Laboratoire de FARCHA (Fort-Lamy, - République du Tchad), 1954 à 1963.

99. Rapport annuel Uganda, 1958, 10.

100. RIJPSTRA ( $A, C$. ), SMIT (A. M.) et SWELLENGREBEL (N. H.) (1961). - How and where to search for the ova of T. saginata. Trop. Geograph. Med. Amsterdam, 13, (2), 161-6.

101. ROBSTER (A. N.) et MALKO (A. T.) (1961). - The marking in med combines of $C$. bovins infected cattle and notification of cases to sanitary-epidemiological stations. Med. Parosit. 1. Parazitorn. Bol. Moscou, 30, (6), 675-7 (en russe).

102. ROLLINSON (D. H. L.) (1960). - Ann. Rep. Dep. Vet. Serv. Anim. Ind., 34.

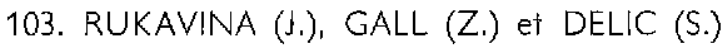
(1962). - Problem teniaze ijudi i Cysticerkoze goveda i svinja u Bosni i Hercegovini. Vet., Sorajevo, 11, (1), 109-116.

104. SCHMID (M.) (1958). - Z Osservazioni sulla frequenza delle Cisticercosi bovina al macello di Trieste. Vet. It., 9. (11), 904-8.

105. SCHULTZE-PETZOLD (H.) (1959). - Wege zu einer wirksamen Bekämpfung der Rinderfinne. Monatsh. f. Trerrheilk., II, (8), 21221.

106. SENS (J.) (1960), - Die U. V. - Lampe bei der Beurteilung von Tieren siammender Lebensmittel. Arch. f. Lebensmittelhyg., 11, 38-9.

107. SGAMBATI (A.) (1959). - La Cisticercosi bovina nelle sue localizzazioni. Zooprofil., 14, (9), 679-87.

108. SILVERMAN (P. H.) (1954 a). - Studies on the biology of some tapeworms of the genus Taenia I. Factors affecting hatching and activation of Taeniid ova and some criteria of their viability. Ann. Trop. Med. Parasit., 48. (2), 207-15. 
109. SILVERMAN (P. H.) (1954). - Studies on the biology of some tapeworms of the genus Taenia Il. The morphology and development of the Taeniid Hexacanth embryo and its enclosing membranes with some notes on the state of development and propagation of gravid segments. Ann. Trop. Med. Porasit., 48, 356-66.

110. SILVERMAN (P. H.) (1955). - A technique for studying the in vitro effect of serum on activated hexacanth embryos. Noture, I76, 598-9.

111. SILVERMAN (P. H.) (1956 o). - The infectivity of the hexacanth embryo of $T$. pisiformis. Trans. Roy. Soc. Trop. Med. Hyg., 50 (1), 7.

112. SILVERMAN (P. H.) (1956 b). - The longevity of eggs of $T$. pisiformis and $T$. saginata under various conditions. Trans. Roy. Soc. Trop. Med. Hyg., 50 (1), 8.

113. SILVERMAN (P. H.) (1956 c). - Specific and non specific in vitro serum reaction to active taeniid hexacanth embryos. Trans. Ray. Soc. Trop. Med. Hyg., 50 (1), 8.

114. SILVERMAN (P. H.) ef HULLAND (T. J.) (1961). - Histological observations on bovine Cysticercosis. Res. Vet. Sci., 2 (3), 24852.

115. Service de Santé de la République du Tchad. Communication personnelle, 1964.

116. SKVORTSOV (A. A.), SOKOLOVA (L. N.) et TALIZIN (F. K.) (1941). - Diagnostic of Cysticercosis in cattle by means of allergic réaction. C. R. Acod. Sci. U.R.S. S., 32 (7). 523-5 (en russe).

117. SOULSBY (E. J. L.) (1963). - Immunological unresponsiveness to Helminth infections in animals. Cong. Int. Med. Vét., Hanovre, 1, 6/A/141, 761-7.

118. THORNTON $(\mathrm{H}$.$) (1957), — Textbook of$ meat inspection-Londres.

119. TRAWINSKI (A.) (1947). - Sur l'emploi de la réaction de précipitation pour le diagnostic de la Cysticercose du porc. Zbl. Bakt., CXXXVI (1/2), 116.

120. TRAWINSKI (A.) (1957). - La Cysticercose chez les animaux et chez l'homme et spécialement la Cysticercose du cerveau. Bull. Off. Int. Epiz., 48, 191-7.
121. TRAWINSKI (A.) (1959). - Diagnostic des maladies parasitaires des moutons provoquées par les vers, à l'aide des méthodes séro-allergiques. Bull. Off. Int. Epiz., 52, 23440.

122. URQUARTH (G. M.) (1958). - The production of experimental Cysticercosis in calves in Kenya. Bull. Epiz. Dis. Afr., 6 (4), 385-93.

123. URQUARTH (G. M.) (1959). - Cysticercosis in cattle and pigs in Africa. Coll. Helm. Anım. Dom. Nairobi. I. A. C.E. D./C. C. T. A., 49, 41-2.

124. URQUARTH (G. M.) (1960), - Diethylcarbamazine therapy in bovine Cysticercosis. J. Parasit., 46 (2), 234.

125. URQUARTH (G. M.) (1961). - Epizootiological and experimental studies on bovine Cysticercosis in East africa. J.1 Parosit., 47 (6), 857-69.

126. VILJOEN (N. F.) (1937). - CysticercosIs in swine and bovines, with special reference to South african conditions. Onderst. J. Vet. ScI. Anim. Ind., 9 (2), 337-570.

127. VINK $(H . H).(1959)$. - Cysticercus inermis bij kalveren. Tijd. V. Diergeneesk., 84 (17), 943-4.

128. VOGE (M.) (1960). - Observations on the structure of Cysticercoids. J. Porasit., 46 (5, Sect. 2), 10.

129. VOGE (M.) (1963). - Observations on the structure of Cysticerci of $T$. solium and $T$. saginata (Cestode: Taeniidae). J. Parasit., 49 (1). 85-90.

130. ZELJKOVIC (S.) et BOKOVIC (T.) (1959). - Istrazivanje bobicavosti kod goveda $\mathrm{i}$ teladi zaklanih u banjaluckoj klanici 1957 godine. Vet. Glasn., Blegrade, 13 (4), 308-10.

N. B. - Pour une biblıgraphie plus étendue ef plus ancienne consulter les index bibliographiques des articles suivants :
I. - VILJOEN (N. F.) (1937).
II. - GRABER (M.) (1959).
III. - LEINATI (L.), MARAZZA (V.), GRIMAL- DI (E.) et PERSIANI (G.) (1963). 


\section{BIBLIOGRAPHIE SUPPLÉMENTAIRE}

1. URQUARTH(G.M.), MC INTHYRE (W.I.M.), MULLIGAN (W.), JARRETT (W. F. H.) and SHORP (N. C. C.) (1963). - Vaccination against Helminth disease. Proc. 17th. World Vet. Congr. Hanovre, 1, 769-774.

2. LIEBMAN (H.) (1963). - Mechanical and biological treatment of sewage for the control of Bovine Cysticercosis. Proc. 17th. World Vet. Congr. Hanovre, 2, 861-866.

3. DAVIDOV (N.K.) (1983). - Economic losses to meat production caused by Helminths in the Uzbek S.S.R. (en russe). Mater. Konf. Probl. Gel'mint. (Samarkand) Uzbek Nauchnoissled. Vet. Inst., 28-30.

4. PELLEGRINI (D.) (1961). - A proposito della localizzazione di Cysticercus bovis. Vet. ital., 12 (2), 140-142.

5. LABUDOVIC (D.) et LUKIC (1963). - Incidence of Cysticercosis in caltle in Serbia (en croate). Vet. Glasn., 17, 869-876.
6. FROYD (G.) (1964 a), - The effect of post infection serum in the infectability of calves with Taenia eggs. Brit. Vet. J., I20 (4), 162166.

7. FROYD (G.) (1964 b). - The longevlty of Cysticercus bovis in bovine tissues. Brit. Vet. J., 120 (5), 205-211.

8. VAN DEN HEEVER (L. W.) and REINECKE (R. K.) (1963). - The significiance of the shoulder incision in the rourine inspection of food animals for Cysticercosis. Proc. 17th World Vet. Congr. Hanovre, 2, 909-912.

9. MC MANUS (D.) (1963), - Prenatal infection of calves with Cysticercus bovis. Vet. Rec., $75(27), 697$.

10. PAVLICEVIC (M.) (1961). - Prilog pozndvanju rasprostranjenosti cisticerkose, Distomatoze, Echinokokoze na Kosovu metohiji. Vet. Glosn., 15 (12), 1023-1026.

11. MIJATOVIC (M:) (1962). - Ikricavost suhog mesa prsula i pastrme. Veterinaria, Sorajevo, II (3), 401-403. 